\title{
ON BOUSFIELD'S PROBLEM FOR SOLVABLE GROUPS OF FINITE PRÜFER RANK
}

\author{
SERGEI O. IVANOV
}

\begin{abstract}
For a group $G$ and $R=\mathbb{Z}, \mathbb{Z} / p, \mathbb{Q}$ we denote by $\hat{G}_{R}$ the $R$-completion of $G$. We study the map $H_{n}(G, K) \rightarrow H_{n}\left(\hat{G}_{R}, K\right)$, where $(R, K)=(\mathbb{Z}, \mathbb{Z} / p),(\mathbb{Z} / p, \mathbb{Z} / p),(\mathbb{Q}, \mathbb{Q})$. We prove that $H_{2}(G, K) \rightarrow H_{2}\left(\hat{G}_{R}, K\right)$ is an epimorphism for a finitely generated solvable group $G$ of finite Prüfer rank. In particular, Bousfield's $H K$-localisation of such groups coincides with the $K$-completion for $K=\mathbb{Z} / p, \mathbb{Q}$. Moreover, we prove that $H_{n}(G, K) \rightarrow H_{n}\left(\hat{G}_{R}, K\right)$ is an epimorphism for any $n$ if $G$ is a finitely presented group of the form $G=M \rtimes C$, where $C$ is the infinite cyclic group and $M$ is a $C$-module.
\end{abstract}

\section{INTRODUCTION}

Throughout the paper $R$ denotes one of the rings $\mathbb{Z}, \mathbb{Q}, \mathbb{Z} / p$ and $K$ denotes one of the fields $\mathbb{Q}$ or $\mathbb{Z} / p$, where $p$ is prime. If $R$ and $K$ occur together, we assume that $K$ is a quotient of $R$. In other words, there are three variants for $(R, K)$ :

$$
(R, K) \in\{(\mathbb{Z}, \mathbb{Z} / p),(\mathbb{Z} / p, \mathbb{Z} / p),(\mathbb{Q}, \mathbb{Q})\} .
$$

Bousfield in [6] introduces the $R$-homological localization $X_{R}$ of a space $X$. It is a space $X_{R}$ with an $R$-homology equivalence $X \rightarrow X_{R}$, which is universal among all $R$-homology equivalences $X \rightarrow Y$. He proves that the $R$-localization exists for any space. The $R$-localization is well understood for 1-connected spaces. However, if $X$ is not 1-connected, even the computation of $\pi_{1}\left(X_{R}\right)$ can be a very difficult problem. Bousfield reduces the problem to a purely algebraic problem introducing the $H R$-localization $G_{H R}$ of a group $G$. It is a group $G_{H R}$ together with a homomorphism $G \rightarrow G_{H R}$ that induces an isomorphism on the level of the first homology $H_{1}(G, R) \cong H_{1}\left(G_{R H}, R\right)$, an epimorphism on the level of the second homology $H_{2}(G, R) \rightarrow H_{2}\left(G_{H R}, R\right)$ and which is universal among such homomorphisms $G \rightarrow H$. He proves that the $H R$-localization exists for any group and

$$
\pi_{1}\left(X_{R}\right)=\pi_{1}(X)_{H R}
$$

The construction of the $H R$-localization is not explicit and it is still difficult to compute it. But we can compare the $H R$-localization with the more explicit construction of $R$-completion of a group $G$, which is defined as follows

$$
\hat{G}_{R}= \begin{cases}\lim _{\longleftarrow} G / \gamma_{i}^{R} G, & \text { if } R \in\{\mathbb{Z}, \mathbb{Z} / p\} \\ \lim _{\longleftarrow} G / \gamma_{i}^{\mathbb{Q}} G \otimes \mathbb{Q}, & \text { if } R=\mathbb{Q},\end{cases}
$$

where $\gamma_{i}^{R} G$ is the lower $R$-central series of $G$ and $-\otimes \mathbb{Q}$ denotes the Malcev completion $(P$ localization, where $P$ is the set of all primes, in terms of [12]) of a nilpotent group. There is

The research is supported by the Russian Science Foundation grant N 16-11-10073. 
a natural homomorphism

$$
G_{H R} \longrightarrow \hat{G}_{R}
$$

If the map (0.1) is an isomorphism, the group $G$ is called $H R$-good (similarly to $R$-good spaces). Otherwise, it is called $H R$-bad. In the book of Bousfield [7] there is only one problem:

Problem (Bousfield): Is there an $H K$-bad finitely presented group for $K=\mathbb{Q}$ or $K=\mathbb{Z} / p$ ?

It is known that a group $G$ is $H R$-good if and only if the map $H_{1}(G, R) \rightarrow H_{1}\left(\hat{G}_{R}, R\right)$ is an isomorphism and $H_{2}(G, R) \rightarrow H_{2}\left(\hat{G}_{R}, R\right)$ is an epimorphism. If $G$ is finitely generated, then $H_{1}(G, R) \rightarrow H_{1}\left(\hat{G}_{R}, R\right)$ is an isomorphism (because $\left.G_{H R} / \gamma_{\omega}^{R}\left(G_{H R}\right)=\hat{G}_{R}[7]\right)$. So the problem of Bousfield can be reformulated as follows: Is there a finitely presented group $G$ such that $H_{2}(G, K) \rightarrow H_{2}\left(\hat{G}_{K}, K\right)$ is not an epimorphism for $K=\mathbb{Q}$ or $K=\mathbb{Z} / p$ ?

We are interested not only in $\hat{G}_{K}$ for $K=\mathbb{Z} / p, \mathbb{Q}$. The pronilpotent completion $\hat{G}_{\mathbb{Z}}$ is interesting as well. It is easy to find an example of an $H \mathbb{Z}$-bad finitely presented group (for example, Klein bottle group $\mathbb{Z} \rtimes C)$. However, the homomorphism on the level of homology with finite coefficients $H_{2}(G, \mathbb{Z} / p) \rightarrow H_{2}\left(\hat{G}_{\mathbb{Z}}, \mathbb{Z} / p\right)$ is usually an epimorphism for all examples that we know. So we generalise the problem of Bousfield and formulate it in the form of conjecture.

Conjecture: If $G$ is a finitely presented group, then the map

$$
H_{2}(G, K) \longrightarrow H_{2}\left(\hat{G}_{R}, K\right)
$$

is an epimorphism for $(R, K) \in\{(\mathbb{Z} / p, \mathbb{Z} / p),(\mathbb{Z}, \mathbb{Z} / p),(\mathbb{Q}, \mathbb{Q})\}$.

In [13] Roman Mikhailov and I proved that the conjecture is true for the class of metabelian groups. Generally we do not believe that the conjecture is true for all finitely presented groups. We tried to find a counterexample among those solvable groups, for which we can compute $H_{2}\left(\hat{G}_{R}, K\right)$. For example, for Abels' group [1] (which is known as a counterexample for some conjectures that are true for metabelian groups) or for finitely presented groups of the form $N \rtimes C$, where $C$ is the infinite cyclic group and $N$ is nilpotent. But in all these cases the map turns out be an epimorphism. Then I found a reason why in all these cases the map is an epmorphism. All these groups are of finite Prüfer rank. The first goal of the paper is to study homological properties of $\hat{G}_{R}$ for solvable groups $G$ of finite Prüfer rank and prove the conjecture for them.

Recall that a group is of type $F_{n}$ if it has a classifying space, whose $n$-skeleton is finite. A group is of type $F_{1}$ if and only if it is finitely generated. A group is of type $F_{2}$ if and only if it is finitely presented. It seems, it is natural to look at the homomorphism $H_{n}(G, K) \rightarrow H_{n}\left(\hat{G}_{R}, K\right)$ for a group $G$ of type $F_{n}$ for any $n$ and ask, when it is an epimorphism. So the most general problem in this direction is the following.

Problem: Describe groups $G$ of type $F_{n}$ such that the map

$$
H_{n}(G, K) \longrightarrow H_{n}\left(\hat{G}_{R}, K\right)
$$

is an epimorphism. 
Bousfield proofs [8] that one of the groups $H_{2}\left(\hat{F}_{\mathbb{Z} / p}, \mathbb{Z} / p\right), H_{3}\left(\hat{F}_{\mathbb{Z} / p}, \mathbb{Z} / p\right)$ is not trivial for a free group $F$, and hence, not for all groups of type $F_{n}$ the map (0.3) is an epimorphism. However, it seems the class of groups of type $F_{n}$ such that the map (0.3) is an epimorphism is very large. We denote by $C$ the infinite cyclic group. If $M$ is a $C$-module, we can consider the semidirect product $M \rtimes C$. Bieri and Strebel describe all modules $M$ such that $M \rtimes C$ is finitely presented [3, Th. C]. It can be shown, and we will show this later, that if $M \rtimes C$ is finitely presented, then $M \rtimes C$ is of type $F_{n}$ for any $n$. The second goal of the article is to prove that for any finitely presented metabelian group of the form $G=M \rtimes C$ the homomorphism (0.3) is an epimorphism for any $n$.

Usually we look at the $R$-completion $\hat{G}_{R}$ as on a discrete group. But it turned out that for our purposes it is useful to look on this group as on a topological group with the topology of inverse limit. Unfortunately, the standard continuous cohomology of topological groups [17] behave well enough only for compact groups. Because of this, we introduce unform cohomology $H_{u}^{*}(\mathscr{G}, K)$ of a topological group $\mathscr{G}$, which are defined similarly to continuous cohomology, but instead of continuous chains we consider uniform chains. Here we consider only topological groups, whose left and right uniform structures coincide. We like the uniform cohomology more then the continuous cohomology because they send inverse limits to direct limits in a more general case (see Proposition 2.6). A topological group $\mathscr{G}$ is said to be $n$-cohomologically discrete over $K$ if the obvious map is an isomorphism

$$
H_{u}^{m}(\mathscr{G}, K) \cong H^{m}(\mathscr{G}, K)
$$

for any $m \leq n$. If a topological group is $n$-cohomologically discrete over $K$ for any $n$, we call it just cohomologically discrete over $K$. This definition is interesting for us because of the following statement that we prove.

Proposition. Let $G$ be a finitely presented group. If $\hat{G}_{R}$ is 2-cohomologically discrete over $K$, then the map (0.2) is an epimorphism.

For more detailed version and the proof see Proposition 4.3.

A group $G$ is of finite Prüfer rank (special rank, reduced rank) if there is a number $r$ such that any finitely generated subgroup of $G$ can be generated by $r$ generators. The minimal $r=r(G)$ with this property is called Prüfer rank of $G$. If there is a short exact sequence of groups $G^{\prime} \nrightarrow G \rightarrow G^{\prime \prime}$, then $G$ is of finite Prüfer rank if and only if $G^{\prime}$ and $G^{\prime \prime}$ are of finite Prüfer rank and $r(G) \leq r\left(G^{\prime}\right)+r\left(G^{\prime \prime}\right)$ [15, Lemma 1.44]. Let $A$ be an abelian group. For prime $p$ we set

$$
{ }_{p} A=\{a \in A \mid p a=0\} .
$$

The group $A$ is of finite Prüfer rank if and only if $A \otimes \mathbb{Q}$ is finite dimensional over $\mathbb{Q},{ }_{p} A$ is finite dimensional over $\mathbb{Z} / p$ and the dimensions are bounded over all $p$ [15, pp. 33-34]. In this case

$$
r(A)=\operatorname{dim}_{\mathbb{Q}}(A \otimes \mathbb{Q})+\max \left\{\operatorname{dim}_{\mathbb{Z} / p}\left({ }_{p} A\right) \mid p \text { is prime }\right\} .
$$

Obviously, a solvable group $G$ is of finite Prüfer rank if and only if there is a finite sequence of normal subgroups $G=U_{1} \supseteq \cdots \supseteq U_{s}=1$ such that $U_{i} / U_{i+1}$ is an abelian group of finite Prüfer rank. For example, the group $\mathbb{Z}[1 / 2] \rtimes C$, where $C=\langle t\rangle$ is the infinite cyclic group acting on $\mathbb{Z}[1 / 2]$ by the multiplication on 2 , is a finitely generated non-polycyclic metabelian group of Prüfer rank 2. We prove the following theorem. 
Theorem. Let $G$ be a solvable group of finite Prüfer rank. Then $\hat{G}_{R}$ is cohomologically discrete over $K$. Moreover, if $G$ is finitely generated, then the map (0.2) is surjective and $G$ is $H K$-good.

For a more detailed version and the proof see Proposition 4.7 and Theorem 4.9.

We denote by $C$ the infinite cyclic group. A finitely generated $C$-module $M$ is called tame if: (1) the torsion subgroup $\operatorname{tor}(M)$ is finite; $(2) M \otimes \mathbb{Q}$ is finite dimensional; (3) there exists a generator $t$ of $C$ such that the characteristic polynomial of $t \otimes \mathbb{Q} \in \operatorname{GL}(M \otimes \mathbb{Q})$ is integral. Bieri and Strebel proved [3, Th. C] that the group $G=M \rtimes C$ is finitely presented if and only if the $C$-module $M$ is tame. Note that a tame $C$-module is of finite Prüfer rank. Hence, $G=M \rtimes C$ is a metabelian group of finite Prüfer rank. It follows from the main theorem of [2] that $G$ is of type $F P_{n}$ for any $n$. By [4] we know that $F P_{n}$ implies $F_{n}$. Therefore, if $M$ is a tame $C$-module, $M \rtimes C$ is of type $F_{n}$ for any $n$. We prove the following theorem.

Theorem. Let $M$ be a tame $C$-module and $G=M \rtimes C$. Then the map (0.3) is an epimorphism for any $n$.

For more detailed version see Proposition 5.7 and Theorem 5.8. Moreover, we prove the following. If $C=\langle t\rangle$ and $M(t-1) \subseteq p M$, then the map (0.3) gives an isomorphism $H_{*}(G, \mathbb{Z} / p) \cong$ $H_{*}\left(\hat{G}_{\mathbb{Z} / p}, \mathbb{Z} / p\right)$ and it follows that the $\mathbb{Z} / p$-localization of the classifying space is the classifying space of the $\mathbb{Z} / p$-completion

$$
(B G)_{\mathbb{Z} / p} \cong B\left(\hat{G}_{\mathbb{Z} / p}\right) .
$$

In particular, the isomorphism (0.4) holds in the case $G=\mathbb{Z}^{n} \rtimes_{a} C$, where $C$ acts on $\mathbb{Z}^{n}$ by a matrix $a \in \mathrm{GL}_{n}(\mathbb{Z})$ such that entries of $a-1$ are divisible by $p$.

A lot of things can be done much simpler here, if we assume that $K \neq \mathbb{Z} / 2 \neq R$ and use the natural isomorphism

$$
H_{*}(A, \mathbb{Z} / p) \cong \Lambda^{*}(A / p) \otimes \Gamma^{* / 2}\left({ }_{p} A\right)
$$

for an abelian group $A$ and $p \neq 2$ (see [9, Ch. V, Th. 6.6], [10]). But we intentionally do not use it because we want to cover the case of $K=\mathbb{Z} / 2$.

The paper is organised as follows. In Section 1 we prove some lemmas that generalise the standard fact that, if a morphism of converging spectral sequences is an isomorphism on some page, then it converges to an isomorphism. In Section 2 we study inverse systems of groups. Namely, we study relations between cohomology of the inverse limit, the direct limit of cohomology and the uniform cohomology of the inverse limit. In section 3 we study mod- $p$ homology of a completion of a solvable group of finite Prüfer rank with respect to any filtration. In Section 4 we study $R$-completions of groups. In particular, we prove here that, if $R$-completion is 2-cohomologically discrete, then the map $H_{2}(G, K) \rightarrow H_{2}\left(\hat{G}_{R}, K\right)$ is an epimorphism, and the main result that the $R$-completion of a solvable group of finite Prüfer rank is cohomologically discrete over $K$. In Section 5 we prove that, for a tame $C$-module $M$, if we set $G=M \rtimes C$, then $H_{n}(G, K) \rightarrow H_{n}\left(\hat{G}_{R}, K\right)$ is an epimorphism.

\section{Lemmas about SPECTRAL SEQUENCES}

Lemma 1.1. Let $f: E \rightarrow E^{\prime}$ be a morphism between two spectral sequences of cohomological type converging to a morphism of graded abelian groups $\varphi: H \rightarrow H^{\prime}$ and $r, n$ be natural numbers. Assume that the spectral sequences are concentrated in the first quadrant. If for 
any pair of integers $(k, l)$ such that $(r-1) k \leq r(n-l)$ the morphism on the level of $r$ th pages $f_{r}^{k, l}: E_{r}^{k, l} \rightarrow E_{r}^{\prime k, l}$ is an isomorphism, then $\varphi^{m}: H^{m} \rightarrow H^{\prime m}$ is an isomorphism for $m \leq n$.

Proof. Fix $n$. Note that for any pair $(k, l)$ such that $k+l \leq n$ and any $r$ the inequality $(r-1) k \leq r(n-l)$ holds. First, assume that $r \geq n+2$. In this case, if $k+l \leq n$, then $E_{r}^{k, l}=E_{\infty}^{k, l}$ and $E_{r}^{\prime k, l}=E_{\infty}^{\prime k, l}$. It follows that $\varphi^{m}$ is an isomorphism for $m \leq n$. Prove now the statement for $r \leq n+2$ by induction on $n+2-r$. We already proved the base step $n+2-r=0$. Prove the inductive step. The direct sum $\bigoplus_{(k, l):(r-1) k \leq r(n-l)} E_{r}^{k, l}$ is a direct summand of the differential module $\left(E_{r}, d_{r}\right)$ because the bidegree of $d_{r}$ is $(r,-r+1)$ and, if $(k, l)$ satisfies $(r-1) k \leq r(n-l)$, then $(k+r, l-r+1)$ satisfies $(r-1)(k+r) \leq r(n-(l-r+1))$. The same for $E^{\prime}$ and $f_{r}: E_{r} \rightarrow E_{r}^{\prime}$ respects this decomposition. It follows that $f_{r}$ induces an isomorphism on $(r+1)$ st page $f_{r+1}^{k, l}: E_{r}^{k, l} \rightarrow E_{r}^{\prime k, l}$ for indexes $(k, l)$ such that $(r-1) k \leq r(n-l)$. In particular, $f_{r+1}^{k, l}$ is an isomorphism for the smaller set of indexes satisfying $((r+1)-1) k \leq(r+1)(n-l)$. The assertion follows from the inductive hypothesis.

There is a dual version. Formally it does not follow from the previous, but the proof is the same.

Lemma 1.2. Let $f: E \rightarrow E^{\prime}$ be a morphism between two spectral sequences of homological type converging to a morphism of graded abelian groups $\varphi: H \rightarrow H^{\prime}$ and $r, n$ be natural numbers. Assume that the spectral sequences are concentrated in the first quadrant. If for any pair of integers $(k, l)$ such that $(r-1) k \leq r(n-l)$ the morphism on the level of rth pages $f_{k, l}^{r}: E_{k, l}^{r} \rightarrow E_{k, l}^{\prime r}$ is an isomorphism, then $\varphi_{m}: H_{m} \rightarrow{H^{\prime}}_{m}$ is an isomorphism for $m \leq n$.

Proof. The proof is the same.

There are two corollaries for the second page.

Corollary 1.3. Let $f: E \rightarrow E^{\prime}$ be a morphism between two spectral sequences of cohomological type converging to a morphism of graded abelian groups $\varphi: H \rightarrow H^{\prime}$ and $n$ be a natural number. Assume that the spectral sequences are concentrated in the first quadrant. If for any pair of integers $(k, l)$ such that $k \leq 2(n-l)$ the morphism $f_{2}^{k, l}: E_{2}^{k, l} \rightarrow E_{2}^{\prime k, l}$ is an isomorphism, then $\varphi^{m}: H^{m} \rightarrow H^{\prime m}$ is an isomorphism for $m \leq n$.

Corollary 1.4. Let $f: E \rightarrow E^{\prime}$ be a morphism between two spectral sequences of homological type converging to a morphism of graded abelian groups $\varphi: H \rightarrow H^{\prime}$ and $n$ be a natural number. Assume that the spectral sequences are concentrated in the first quadrant. If for any pair of integers $(k, l)$ such that $k \leq 2(n-l)$ the morphism $f_{k, l}^{2}: E_{k, l}^{2} \rightarrow E_{k, l}^{\prime 2}$ is an isomorphism, then $\varphi_{m}: H_{m} \rightarrow H^{\prime}{ }_{m}$ is an isomorphism for $m \leq n$.

\section{Cohomology of inverse limits}

Throughout the section we denote by $P$ a fixed filtered partially ordered set (=directed set). The word "filtered" means that for any $i, j \in P$ there exists $k \in P$ such that $k \geq i$ and $k \geq j$. The main example of $P$ for us is $\mathbb{N}$ but occasionally we use other examples like all finitely generated subgroups of a group. By a directed system in a category $\mathfrak{C}$ we mean a functor $\mathcal{C}: P \rightarrow \mathfrak{C}$, where $P$ is treated as a category. For $i \in P$ we set $C^{i}:=\mathcal{C}(i)$. Dually we define an inverse system $\mathcal{C}: P^{\text {op }} \rightarrow \mathfrak{C}$. In the case of inverse systems we use the dual notation $C_{i}:=\mathcal{C}(i)$. We say that a property holds for big enough $i$ if there exists $k \in P$ such that the property holds for any $i \geq k$. 
We use directed limits (=filtered colimits) lim of directed systems and inverse limits lim of inverse systems. The important advantage of directed limits in contrast to inverse limits is that $\lim _{\longrightarrow}: \mathrm{Ab}^{P} \rightarrow \mathrm{Ab}$ is an exact functor.

Throughout the section $\mathcal{G}$ denotes an inverse system groups and epimorphims. We set $G_{i}:=\mathcal{G}(i)$. So we assume that the homomorphism $G_{j} \rightarrow G_{i}$ is an epimorphism for any $i \leq j$.

Let $S$ be a commutative ring. A direct $S[\mathcal{G}]$-module $\mathcal{M}$ is a direct system of abelian groups, together with a structure of $S\left[G_{i}\right]$-module on $M^{i}$ such that $M^{i} \rightarrow M^{j}$ is a homomorphism of $S\left[G_{j}\right]$-modules for any $i \leq j$. In the case $S=\mathbb{Z}$ we call them just direct $\mathcal{G}$-modules. If we set

$$
\hat{\mathcal{G}}:=\lim _{\longleftarrow} G_{i}, \quad \breve{\mathcal{M}}=\underline{\lim _{\longrightarrow}} M^{i},
$$

then $\breve{\mathcal{M}}$ has a natural structure of a $S[\hat{\mathcal{G}}]$-module. Note that if $\mathcal{G}^{\prime} \nrightarrow \mathcal{G} \rightarrow \mathcal{G}^{\prime \prime}$ is a short exact sequence of groups and epimorphisms, then by Mittag-Leffler condition the sequence of inverse limits $\hat{\mathcal{G}}^{\prime} \nrightarrow \hat{\mathcal{G}} \rightarrow \hat{\mathcal{G}}^{\prime \prime}$ is short exact.

2.1. Cohomologically discrete direct modules. A direct $\mathcal{G}$-module $\mathcal{M}$ is said to be $n$ cohomologically discrete, if the homomorphism

$$
\stackrel{\lim _{\longrightarrow}}{\longrightarrow} H^{m}\left(G_{i}, M^{i}\right) \longrightarrow H^{m}(\hat{\mathcal{G}}, \breve{\mathcal{M}})
$$

is an isomorphism for any $m \leq n$ (further we will prove that this definition is equivalent to a definition given on the language of uniform cohomology for some class of direct modules Proposition 2.7). The inverse system $\mathcal{G}$ is said to be $n$-cohomologically discrete over $K$ if the constant $\mathcal{G}$-module $K$ is $n$-cohomologically discrete. If $\mathcal{G}$ is $n$-cohomologically discrete $K$ for any $n$, it is called cohomologically discrete over $K$.

Proposition 2.1. Let $\mathcal{G}^{\prime} \nrightarrow \mathcal{G} \rightarrow \mathcal{G}^{\prime \prime}$ be a short exact sequence of inverse systems of groups and epimorphisms and $\mathcal{M}$ be a direct $\mathcal{G}$-module. Assume that

(1) $\mathcal{M}$ is n-cohomologically discrete as a direct $\mathcal{G}^{\prime}$-module;

(2) the direct $\mathcal{G}^{\prime \prime}$-module $H^{m}\left(\mathcal{G}^{\prime}, \mathcal{M}\right)=\left\{H^{m}\left(G_{i}^{\prime}, M^{i}\right)\right\}$ is $2(n-m)$-cohomologically discrete for any $0 \leq m \leq n$.

Then $\mathcal{M}$ is $n$-cohomologically discrete as a direct $\mathcal{G}$-module.

Proof. For any fixed $i$ the morphism of short exact sequences

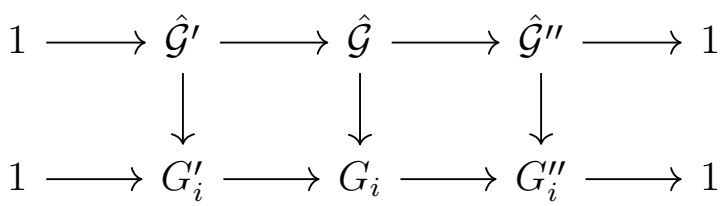

and the homomorphism of $\hat{\mathcal{G}}$-modules $\hat{\mathcal{M}} \rightarrow M^{i}$ yield a morphism of Lyndon-Hochschild-Serre spectral sequences, whose second page component is

$$
H^{*}\left(G_{i}^{\prime \prime}, H^{*}\left(G_{i}^{\prime}, M^{i}\right)\right) \longrightarrow H^{*}\left(\hat{\mathcal{G}}^{\prime \prime}, H^{*}\left(\hat{\mathcal{G}}^{\prime}, \breve{M}\right)\right)
$$

and that converges to the morphism $H^{*}\left(G_{i}, M^{i}\right) \rightarrow H^{*}(\hat{\mathcal{G}}, \breve{\mathcal{M}})$. On the left hand we have a direct system of spectral sequences. Since direct limit is an exact functor, we obtain the spectral sequence of direct limits, whose second page is $\lim _{i} H^{*}\left(G_{i}^{\prime \prime}, H^{*}\left(G_{i}^{\prime}, M^{i}\right)\right)$ and that converges to $\lim H^{*}\left(G_{i}, M^{i}\right)$. Moreover, we obtain a morphism of spectral sequences, whose second page component is

$$
\lim _{\longrightarrow} H^{*}\left(G_{i}^{\prime \prime}, H^{*}\left(G_{i}^{\prime}, M^{i}\right)\right) \longrightarrow H^{*}\left(\hat{\mathcal{G}}^{\prime \prime}, H^{*}\left(\hat{\mathcal{G}}^{\prime}, \breve{\mathcal{M}}\right)\right)
$$


and that converges to the homomorphism $\lim _{\longrightarrow} H^{*}\left(G_{i}, M^{i}\right) \rightarrow H^{*}(\hat{\mathcal{G}}, \breve{\mathcal{M}})$. Assume that $(k, l)$ is a pair of non negative integers such that $k \leq 2(n-l)$. Then

$$
\stackrel{\lim _{\longrightarrow}}{\longrightarrow} H^{k}\left(G_{i}^{\prime \prime}, H^{l}\left(G_{i}^{\prime}, M^{i}\right)\right) \longrightarrow H^{k}\left(\hat{\mathcal{G}}^{\prime \prime}, \lim _{\longrightarrow} H^{l}\left(G_{i}^{\prime}, M^{i}\right)\right)
$$

is an isomorphism because $H^{l}\left(\mathcal{G}^{\prime}, \mathcal{M}\right)$ is $2(n-l)$-cohomologically discrete. The map $\lim _{\longrightarrow} H^{l}\left(G_{i}^{\prime}, M^{i}\right) \rightarrow H^{l}\left(\hat{\mathcal{G}}^{\prime}, \breve{\mathcal{M}}\right)$ is an isomorphism because $\mathcal{M}$ is $n$-homologically discrete as $\overrightarrow{\text { a direct }} \mathcal{G}^{\prime}$-module. It follows that

$$
\lim _{\longrightarrow} H^{k}\left(G_{i}^{\prime \prime}, H^{l}\left(G_{i}^{\prime}, M^{i}\right)\right) \longrightarrow H^{k}\left(\hat{\mathcal{G}}^{\prime \prime}, H^{l}\left(\hat{\mathcal{G}}^{\prime}, \breve{\mathcal{M}}\right)\right)
$$

is an isomorphism for pairs $(k, l)$ such that $k \leq 2(n-l)$. The assertion follows from Corollary 1.3 .

Corollary 2.2. Let $\mathcal{G}^{\prime} \rightarrow \mathcal{G} \rightarrow \mathcal{G}^{\prime \prime}$ be a short exact sequence of inverse systems of groups and epimorphisms. Assume that

(1) $\mathcal{G}^{\prime}$ is $n$-cohomologically discrete over $K$;

(2) the direct $\mathcal{G}^{\prime \prime}$-module $H^{m}\left(\mathcal{G}^{\prime}, K\right)=\left\{H^{m}\left(G_{i}^{\prime}, K\right)\right\}$ is $2(n-m)$-cohomologically discrete for any $0 \leq m \leq n$.

Then $\mathcal{G}$ is n-cohomologically discrete.

2.2. Quasiconstant direct systems. Let $\mathfrak{A}$ be an abelian category. A direct system $\mathcal{A}$ in $\mathfrak{A}$ is said to be zero-equivalent if for any $i$ there is $j>i$ such that the morphism $A^{i} \rightarrow A^{j}$ vanishes. In this case $\underset{\lim }{\longrightarrow} A^{i}$ exists and vanishes.

Let $\mathcal{A}$ be a direct system in $\mathfrak{A}$. Assume that $\breve{\mathcal{A}}=\underset{\lim }{\longrightarrow} A^{i}$ exists. Consider the kernels $B^{i}=$ $\operatorname{Ker}\left(A^{i} \rightarrow \breve{\mathcal{A}}\right)$. They form a direct system of groups $\mathcal{B}$. Then $\mathcal{A}$ is called quasiconstant if $\mathcal{B}$ is zero-equivalent and the morphism $A^{i} \rightarrow \breve{\mathcal{A}}$ is an epimorphism for big enough $i$. Equivalently, $\mathcal{A}$ is quasiconstant if there exists a short exact sequence of direct systems $\mathcal{B} \rightarrow \mathcal{A} \rightarrow \mathcal{C}$ such that $\mathcal{B}$ is zero-equivalent and $C^{i} \rightarrow C^{j}$ is an isomorphism for big enough $i$ and any $j \geq i$. In this case $\breve{A} \cong C^{i}$ for big enough $i$.

Dually one can define zero-equivalent inverse systems and quasiconstant inverse systems.

Lemma 2.3. Let $\mathfrak{F}: \mathfrak{A}^{\mathrm{op}} \rightarrow \mathfrak{B}$ be an exact contravariant functor from an abelian category $\mathfrak{A}$ to an abelian category $\mathfrak{B}$. If $\mathcal{A}$ is a quasiconstant inverse system in $\mathfrak{A}$, then $\mathfrak{F}(\mathcal{A})$ is a quasiconstant direct system in $\mathfrak{B}$ and

$$
\underset{\lim }{\longrightarrow} \mathfrak{F}\left(A_{i}\right)=\mathfrak{F}\left(\lim _{\longleftarrow} A_{i}\right) .
$$

Proof. Obvious.

Lemma 2.4. Let $\mathcal{A}$ be an inverse system (resp. directed system) of $S$-modules of finite length over $S$. Assume that the lengths of the modules are bounded above i.e. there exists $n$ such that length $\left(A_{i}\right) \leq n$ (resp. length $\left(A^{i}\right) \leq n$ ) for any $i$. Then $\mathcal{A}$ is quasiconstant and length $\left(\lim _{\longleftarrow} A_{i}\right) \leq n$.

Proof. For any fixed $i$ the system of images $\operatorname{Im}\left(A_{j} \rightarrow A_{i}\right)$ for $j \geq i$ stabilises because $A_{i}$ has finite length. In other words there exists $C_{i} \subseteq A_{i}$ such that $C_{i}=\operatorname{Im}\left(A_{j} \rightarrow A_{i}\right)$ for big enough $j \geq i$. Note that the inverse system $A_{i} / C_{i}$ is zero-equivalent. It is easy to see that $C_{i+1} \rightarrow C_{i}$ is an epimorphism. Hence, length $\left(C_{j}\right) \geq$ length $\left(C_{i}\right)$ for $j \geq i$. Since the lengths are bounded above, $C_{j} \rightarrow C_{i}$ is an isomorphism for big enough $i$.

The proof for directed systems is similar. 
A direct $S[\mathcal{G}]$-module is called quasiconstant if it is qasiconstant as a direct system of abelian groups.

Lemma 2.5. Let $\mathcal{M}$ be a direct $S[\mathcal{G}]$-module such that each $M^{i}$ is a Noetherian $S\left[G_{i}\right]$-module. Then the following are equivalent.

(1) $\breve{\mathcal{M}}$ is a Noetherian $S[\hat{\mathcal{G}}]$-module.

(2) $\mathcal{M}$ is quasiconstant.

Proof. $(2) \Rightarrow(1)$ is obvious because $\breve{\mathcal{M}}$ is a quotient of $M^{i}$ for big enough $i$.

$(1) \Rightarrow(2)$. Since $\breve{\mathcal{M}}=\bigcup_{i=1}^{\infty} \operatorname{Im}\left(M^{i} \rightarrow \breve{\mathcal{M}}\right)$ and $\breve{\mathcal{M}}$ is Noetherian, we obtain $\operatorname{Im}\left(M^{i} \rightarrow \breve{\mathcal{M}}\right)=\breve{M}$ for big enough $i$. Hence $M^{i} \rightarrow \breve{\mathcal{M}}$ is an epimorphism for big enough $i$. Without loss of generality we can further assume that it is an epimorphism for all $i$. Set $N^{i}=\operatorname{Ker}\left(M^{i} \rightarrow \breve{\mathcal{M}}\right)$. Then we have a short exact sequence of direct modules $\mathcal{N} \rightarrow \mathcal{M} \rightarrow \breve{\mathcal{M}}$. It follows that $\lim _{\longrightarrow} N^{i}=0$. Hence for any fixed $i$ we have $\bigcup_{j>i}^{\infty} \operatorname{Ker}\left(N^{i} \rightarrow N^{j}\right)=N^{i}$. Using that $M^{i}$ is Noetherian, we obtain $N^{i}=\operatorname{Ker}\left(N^{i} \rightarrow N^{j}\right)$ for big enough $j$.

2.3. Cohomologically discrete inverse limits. Let $\mathscr{G}$ be a topological group, whose right and left uniform structures coincide. For a topological $\mathscr{G}$-module $\mathscr{M}$ we define uniform cohomology of $\mathscr{G}$ with coefficients in $\mathscr{M}$

$$
H_{u}^{n}(\mathscr{G}, \mathscr{M})
$$

similarly to continuous cohomology [17] but instead of the complex of continuous cochains we consider the complex of uniform cochains

$$
C_{u}^{n}(\mathscr{G}, \mathscr{M})=\left\{f: \mathscr{G}^{n} \rightarrow \mathscr{M} \mid f \text { is uniform }\right\} .
$$

If $\mathscr{G}$ is a compact topological group, uniform cohomology coincide with continuous cohomology.

A discrete $\mathscr{G}$-module $\mathscr{M}$ is said to be $n$-cohomologically discrete if the morphism

$$
H_{u}^{m}(\mathscr{G}, \mathscr{M}) \longrightarrow H^{m}(\mathscr{G}, \mathscr{M})
$$

is an isomorphism for $m \leq n$. The topological group $\mathscr{A}$ is said to be $n$-cohomologically discrete over a commutative ring $S$ if $S$ is a $n$-cohomologically discrete $\mathscr{G}$-module.

Proposition 2.6. Let $\mathcal{M}$ be a quasiconstant direct $\mathcal{G}$-module. Consider $\hat{\mathcal{G}}$ as a topological group with the topology of inverse limit and $\breve{\mathcal{M}}$ as a discrete $\hat{\mathcal{G}}$-module. Then

$$
H_{u}^{*}(\hat{\mathcal{G}}, \breve{\mathcal{M}})=\lim _{\longrightarrow} H^{*}\left(G_{i}, M^{i}\right) .
$$

Proof. Without loss of generality, we can assume that $M^{i} \rightarrow \breve{M}$ is an epimorphism for any $i$. Set $N^{i}:=\operatorname{Ker}\left(M^{i} \rightarrow \breve{M}\right)$. The short exact sequence $N^{i} \rightarrow M^{i} \rightarrow \breve{\mathcal{M}}$ gives rise a long exact sequence

$$
H^{n-1}\left(G_{i}, N^{i}\right) \rightarrow H^{n}\left(G_{i}, \breve{\mathcal{M}}\right) \rightarrow H^{n}\left(G_{i}, M^{i}\right) \rightarrow H^{n}\left(G_{i}, N^{i}\right) .
$$

Since $\mathcal{N}$ is zero-equivalent, $H^{*}\left(G_{i}, N_{i}\right)$ is zero-equivalent as well. Since the functor of direct limit is exact, it follows that $\lim _{\longrightarrow} H^{n}\left(G_{i}, \breve{\mathcal{M}}\right) \cong \lim _{\longrightarrow} H^{n}\left(G_{i}, M^{i}\right)$.

Now we need to prove that $H^{*}(\hat{\mathcal{G}}, \breve{\mathcal{M}})=\lim H^{*}\left(G_{i}, \breve{\mathcal{M}}\right)$. Since the functor of direct limit is exact, it is enough to prove that $C_{u}^{n}(\hat{\mathcal{G}}, \overrightarrow{\mathcal{M}})=\underset{\longrightarrow}{\longrightarrow} C^{n}\left(G_{i}, \breve{\mathcal{M}}\right)$. Consider the morphism $\lim _{\longrightarrow} C^{n}\left(G_{i}, \breve{\mathcal{M}}\right) \rightarrow C_{u}^{n}(\hat{\mathcal{G}}, \breve{\mathcal{M}})$. Since the maps $\hat{\mathcal{G}} \rightarrow G_{i}$ are epimorphisms, we get that it is $\vec{a}$ monomorphism. In order to prove that it is an epimorphism, we need to prove that for any $f \in C_{u}^{n}(\hat{\mathcal{G}}, \breve{\mathcal{M}})$ there exists $i$ and $\bar{f} \in C^{n}\left(G_{i}, \breve{\mathcal{M}}\right)$ such that $f$ is a composition of $\bar{f}$ 
and the projection $\hat{\mathcal{G}}^{n} \rightarrow G_{i}^{n}$. Set $H_{i}:=\operatorname{Ker}\left(\hat{\mathcal{G}} \rightarrow G_{i}\right)$. Then the uniform structure on $\hat{\mathcal{G}}$ is generated by sets $U_{i}=\left\{\left(g, g^{\prime}\right) \mid g H_{i}=g^{\prime} H_{i}\right\}$. It follows that for any $f$ there exists $i$ such that $f\left(g_{1}, \ldots, g_{n}\right)=f\left(g_{1}^{\prime}, \ldots, g_{n}^{\prime}\right)$ whenever $g_{k} H_{i}=g_{k}^{\prime} H_{i}$ for any $k$. Then, if we identify $G_{i}$ with $\hat{\mathcal{G}} / H_{i}$, we can define $\bar{f}\left(g_{1} H_{i}, \ldots, g_{n} H_{i}\right)=f\left(g_{1}, \ldots, g_{n}\right)$.

Corollary 2.7. Let $\mathcal{M}$ be a quasiconstant direct $\mathcal{G}$-module. Then $\mathcal{M}$ is $n$-cohomologically discrete as a direct $\mathcal{G}$-module if and only if $\breve{\mathcal{M}}$ is $n$-cohomologically discrete as a discrete $\hat{\mathcal{G}}$-module.

Proposition 2.8. Let $\mathcal{G}^{\prime} \rightarrow \mathcal{G} \rightarrow \mathcal{G}^{\prime \prime}$ be a short exact sequence of inverse systems of groups and epimorphisms and $\mathcal{M}$ be a quasiconstant direct $\mathcal{G}$-module. Assume that

(1) $\breve{\mathcal{M}}$ is $n$-cohomologically discrete as a discrete $\hat{\mathcal{G}}^{\prime}$-module;

(2) the discrete $\hat{\mathcal{G}}^{\prime \prime}$-module $H^{m}\left(\hat{\mathcal{G}}^{\prime}, \breve{\mathcal{M}}\right)$ is $2(n-m)$-cohomologically discrete for any $0 \leq m \leq n$.

(3) the direct system $H^{m}\left(G_{i}^{\prime}, M^{i}\right)$ is quasiconstant for any $0 \leq m \leq n$.

Then $\breve{\mathcal{M}}$ is $n$-cohomologically discrete as a discrete $\hat{\mathcal{G}}$-module.

Proof. By Corollary 2.7 we obtain that $\mathcal{M}$ is $n$-cohomologically discrete as a direct $\mathcal{G}^{\prime}$-module. Then by Proposition 2.1 and the Corollary 2.7 it is enough to prove that $\left\{H^{m}\left(G_{i}^{\prime}, M^{i}\right)\right\}$ is $2(n-m)$-cohomologically discrete direct $\mathcal{G}^{\prime \prime}$-module for any $0 \leq m \leq n$. Fix $k \leq 2(n-m)$. Then we need to prove that $\lim _{\longrightarrow} H^{k}\left(G_{i}^{\prime \prime}, H^{m}\left(G_{i}^{\prime}, M^{i}\right)\right) \cong H^{k}\left(\hat{\mathcal{G}}^{\prime \prime}, \lim _{\longrightarrow} H^{m}\left(G_{i}^{\prime}, M^{i}\right)\right)$. We have

$$
\lim _{\longrightarrow} H^{k}\left(G_{i}^{\prime \prime}, H^{m}\left(G_{i}^{\prime}, M^{i}\right)\right) \cong H_{u}^{k}\left(\hat{\mathcal{G}}^{\prime \prime}, \lim _{\longrightarrow} H^{m}\left(G_{i}^{\prime}, M^{i}\right)\right)
$$

because $H^{m}\left(G_{i}^{\prime}, M^{i}\right)$ is quasiconstant. We have

$$
H_{u}^{k}\left(\hat{\mathcal{G}}^{\prime \prime}, \lim _{\longrightarrow} H^{m}\left(G_{i}^{\prime}, M^{i}\right)\right) \cong H_{u}^{k}\left(\hat{\mathcal{G}}^{\prime \prime}, H^{m}\left(\hat{\mathcal{G}}^{\prime}, \breve{\mathcal{M}}\right)\right)
$$

because $\mathcal{M}$ is $n$-cohomologically discrete over $\mathcal{G}^{\prime}$. We have

$$
H_{u}^{k}\left(\hat{\mathcal{G}}^{\prime \prime}, H^{m}\left(\hat{\mathcal{G}}^{\prime}, \breve{\mathcal{M}}\right)\right) \cong H^{k}\left(\hat{\mathcal{G}}^{\prime \prime}, H^{m}\left(\hat{\mathcal{G}}^{\prime}, \breve{\mathcal{M}}\right)\right)
$$

because $H^{m}\left(\hat{\mathcal{G}}^{\prime}, \breve{\mathcal{M}}\right)$ is $2(n-m)$-cohomologically discrete as a discrete $\hat{\mathcal{G}}^{\prime \prime}$-module. And using again that $\mathcal{M}$ is $n$-cohomologically discrete over $\mathcal{G}^{\prime}$ we get

$$
H^{k}\left(\hat{\mathcal{G}}^{\prime \prime}, H^{m}\left(\hat{\mathcal{G}}^{\prime}, \breve{\mathcal{M}}\right)\right) \cong H^{k}\left(\hat{\mathcal{G}}^{\prime \prime}, \lim _{\longrightarrow} H^{m}\left(G_{i}^{\prime}, M^{i}\right)\right) .
$$

Composing these isomorphisms we obtain that $\left\{H^{m}\left(G_{i}^{\prime}, M^{i}\right)\right\}$ is $2(n-m)$-cohomologically discrete direct $\mathcal{G}^{\prime \prime}$-module for any $0 \leq m \leq n$.

\section{Mod-P homology of COMPletions of ABElian AND SOlVABle GROUPS OF FINITE PRÜFER RANK}

For an abelian group $A$ we set

$$
\begin{gathered}
{ }_{n} A=\{a \in A \mid n a=0\}, \quad{ }_{(n)} A=\bigcup_{i=1}^{\infty} n^{i} A, \\
\operatorname{tor}(A)=\bigcup_{n=1}^{\infty}{ }_{n} A, \quad A / n=A / n A .
\end{gathered}
$$

We freely use that for a torsion free abelian group $A$ there are isomorphisms $H_{*}(A) \cong \Lambda^{*}(A)$ and $H_{*}(A, \mathbb{Z} / p) \cong \Lambda^{*}(A / p)$. If we set $V^{\vee}=\operatorname{Hom}_{\mathbb{Z} / p}(V, \mathbb{Z} / p)$, then $H^{*}\left(A, M^{\vee}\right)=H_{*}(A, M)^{\vee}$ for a $\mathbb{Z} / p[A]$-module $M$. If $M$ is finite, $\left(M^{\vee}\right)^{\vee}=M$. Then for a finite $\mathbb{Z} / p[A]$-module there is an 
isomorphism $H^{*}(A, M)=H_{*}\left(A, M^{\vee}\right)^{\vee}$. Using this, one can obtain cohomological versions of all homological statements in the section.

\subsection{Lemmas about abelian groups.}

Lemma 3.1. Let $f: A \rightarrow B$ be a homomorphism of abelian groups.

(1) If $f$ induces isomorphisms $A / p \cong B / p$ and ${ }_{p} A \cong{ }_{p} B$, then it induces an isomorphism $H_{*}(A, \mathbb{Z} / p) \cong H_{*}(B, \mathbb{Z} / p)$.

(2) If $f$ induces trivial maps $A / p \stackrel{0}{\rightarrow} B / p$ and ${ }_{p} A \stackrel{0}{\rightarrow}{ }_{p} B$, then the $\operatorname{map}_{n}(A, \mathbb{Z} / p) \stackrel{0}{\rightarrow}$ $H_{n}(B, \mathbb{Z} / p)$ is trivial for $n \geq 1$.

Proof. (1) First assume that $B=0$ and prove that $H_{n}(A, \mathbb{Z} / p)=0$ for $n \geq 1$. Consider the torsion subgroup tor $(A) \subseteq A$ and the torsion free quotient $\operatorname{tf}(A)=A / \operatorname{tor}(A)$. Then $\operatorname{tf}(A) / p=0$, and hence, $H_{*}(\operatorname{tf}(A), \mathbb{Z} / p)=\Lambda^{*}(\operatorname{tf}(A) / p)=0$. Since ${ }_{p} A=0$, we have ${ }_{(p)} A=0$, and then $\operatorname{tor}(A)$ consists of non- $p$-torsion. Then $H_{n}(\operatorname{tor}(A), \mathbb{Z} / p)=0$. Then the second page of the spectral sequence $H_{*}\left(\operatorname{tf}(A), H_{*}(\operatorname{tor}(A), \mathbb{Z} / p)\right)$ consists of zeros except the position $(0,0)$.

If $f$ is a monomorphism, the cokernel $D$ has the properties ${ }_{p} D=0$ and $D / p=0$ because of the exact sequence $0 \rightarrow{ }_{p} A \rightarrow{ }_{p} B \rightarrow{ }_{p} D \rightarrow A / p \rightarrow B / p \rightarrow D / p \rightarrow 0$. Then $H_{n}(D, \mathbb{Z} / p)=0$ for $n \geq 1$, and using the spectral sequence $H_{*}\left(D, H_{*}(A, \mathbb{Z} / p)\right) \Rightarrow H_{*}(B, \mathbb{Z} / p)$ we obtain the result.

The proof is similar if $f$ is an epimorphism.

Consider the general case. Denote by $I$ the image of $f$. Since $f$ induces isomorphisms ${ }_{p} A \cong{ }_{p} B$ and $A / p \cong B / p$, the maps ${ }_{p} A \rightarrow{ }_{p} I$ and $A / p \rightarrow I / p$ are monomorphisms and the maps ${ }_{p} I \rightarrow{ }_{p} B$ and $I / p \rightarrow B / p$ are epimorphisms. Using that $-\otimes \mathbb{Z} / p$ is right exact and $\operatorname{Tor}(-, \mathbb{Z} / p)$ is left exact, we obtain that $A / p \rightarrow I / p$ and ${ }_{p} I \rightarrow{ }_{p} B$ are isomorphisms. Then all these four maps are isomorphisms and, using that we proved above, we get $H_{*}(A, \mathbb{Z} / p) \cong H_{*}(I, \mathbb{Z} / p) \cong$ $H_{*}(B, \mathbb{Z} / p)$.

(2) Denote by $P$ the set of couples of finitely generated abelian groups $\left(A^{\prime}, B^{\prime}\right)$ such that $A^{\prime} \leq A, B^{\prime} \leq B$ and $f\left(A^{\prime}\right) \leq p B^{\prime}$. Consider $P$ with the natural filtered order. Obviously we have $B=\bigcup_{\left(A^{\prime}, B^{\prime}\right) \in P} B^{\prime}$. Prove that $A=\bigcup_{\left(A^{\prime}, B^{\prime}\right) \in P} A^{\prime}$. Indeed, for any finitely generated subgroup $A^{\prime} \leq A$ generated by $a_{1}, \ldots, a_{m}$ we have $f\left(a_{i}\right) \in p B$ because the map $A / p \rightarrow B / p$ is trivial. Hence, we can find $b_{1}, \ldots, b_{m} \in B$ such that $p b_{i}=f\left(a_{i}\right)$ and consider the subgroup $B^{\prime}$ generated by them. Therefore $\left(A^{\prime}, B^{\prime}\right) \in P$ and $A=\bigcup_{\left(A^{\prime}, B^{\prime}\right) \in P} A^{\prime}$. Then $A=\lim _{\left(A^{\prime}, B^{\prime}\right) \in P} A^{\prime}$ and $B=\lim _{\left(A^{\prime}, B^{\prime}\right) \in P} B^{\prime}$. So, using that homology commute with direct limits, we deduce the statement to finitely generated abelian groups.

Prove the statement for finitely generated abelian groups. Using the functorial isomorphism $H_{1}(A, \mathbb{Z} / p)=A / p$ we obtain the result for $n=1$. Prove it for $n \geq 2$. Using the Künneth formula, we obtain that it is enough to prove this for three cases $A=\mathbb{Z}, A=\mathbb{Z} / p^{k}$ and $A=\mathbb{Z} / q^{k}$ for $q \neq p$. For $A=\mathbb{Z} / q^{k}$ and $A=\mathbb{Z}$ it is obvious because $H_{n}\left(\mathbb{Z} / q^{k}, \mathbb{Z} / p\right)=0$ and $H_{n}(\mathbb{Z}, \mathbb{Z} / p)$ for $n \geq 2$.

Consider the case $A=\mathbb{Z} / p^{k}$. The assumptions on $f$ imply that $f$ factors through the homomorphism $\varphi: \mathbb{Z} / p^{k} \rightarrow \mathbb{Z} / p^{k}$ of multiplication by $p: \varphi(a)=p a$. Then it is enough to prove that $\varphi_{*}: H_{n}\left(\mathbb{Z} / p^{k}, \mathbb{Z} / p\right) \rightarrow H_{n}\left(\mathbb{Z} / p^{k}, \mathbb{Z} / p\right)$ is trivial for $n \geq 2$. Consider the functorial short exact sequence $\Lambda^{2}(A / p) \nrightarrow H_{2}(A, \mathbb{Z} / p) \rightarrow{ }_{p} A$. Using that $\Lambda^{2}(\mathbb{Z} / p)=0$, that $\varphi$ induces the trivial $\operatorname{map}_{p}\left(\mathbb{Z} / p^{k}\right) \rightarrow{ }_{p}\left(\mathbb{Z} / p^{k}\right)$ and that the sequence is functoral, we obtain the result for $n=2$. Since homology in our case are dual to cohomology, it is enough to prove for cohomology. The map $\varphi: H^{*}\left(\mathbb{Z} / p^{k}, \mathbb{Z} / p\right) \rightarrow H^{*}\left(\mathbb{Z} / p^{k}, \mathbb{Z} / p\right)$ is an algebra homomorphism. For odd $p$ or $p=2$ and $k>1$ we have isomorphism of algebras $H^{\star}\left(\mathbb{Z} / p^{k}, \mathbb{Z} / p\right) \cong \mathbb{Z} / p[x, y] /\left(x^{2}\right)$, and for $p=2$ for $k=1$ we have $H^{*}(\mathbb{Z} / 2, \mathbb{Z} / 2) \cong \mathbb{Z} / 2[x]$, where $|x|=1$ and $|y|=2$. Then $H^{*}\left(\mathbb{Z} / p^{k}, \mathbb{Z} / p\right)$ is generated in 
the first and the second degree. Then, using that the maps $H^{n}\left(\mathbb{Z} / p^{k}, \mathbb{Z} / p\right) \rightarrow H^{n}\left(\mathbb{Z} / p^{k}, \mathbb{Z} / p\right)$ are trivial for $n=1,2$, we obtain that they are trivial for all $n$.

Lemma 3.2. Let $A_{1} \leftarrow A_{2} \leftarrow \ldots$ be an inverse sequence of abelian groups and epimorphisms such that $A_{i} / p$ is finite for any $i$. Then the obvious maps are isomorphisms

$$
{ }_{p}\left(\lim _{\longleftarrow} A_{i}\right) \cong \lim _{\longleftarrow}\left({ }_{p} A_{i}\right), \quad\left(\lim _{\longleftarrow} A_{i}\right) / p \cong \lim _{\longleftarrow}\left(A_{i} / p\right) .
$$

Proof. It follows from [13, Prop. 2.4.] and the Mittag-Leffler condition.

Lemma 3.3. Let $A$ be an abelian group such that $A / p$ is finite for any $p$ and $A \supseteq A_{1} \supseteq A_{2} \supseteq$.. be a sequence of subgroups. Set $\hat{A}=\lim _{\longrightarrow} A / A_{i}$ and $\hat{A}_{i}=\operatorname{Ker}\left(\hat{A} \rightarrow A / A_{i}\right)$. Then for any $n$ there exists $i$ such that

$$
\hat{A}_{i} \subseteq n \hat{A} .
$$

Proof. First we prove it for prime $n=p$. By Lemma 3.2 we get that $\hat{A} / p=\underline{\lim }_{i}\left(A / A_{i}\right) / p$. Moreover, $\operatorname{dim}\left(\left(A / A_{i}\right) / p\right) \leq \operatorname{dim}(A / p)$, and hence, the sequence stabilises: $\left(A / A_{i}\right) / p=\left(\hat{A} / \hat{A}_{i}\right) / p=$ $\hat{A} / p$ for big enough $i$. Thus $p \hat{A}=p \hat{A}+\hat{A}_{i}$ for big enough $i$. It follows that for any $p$ there is $i$ such that $\hat{A}_{i} \subseteq p \hat{A}$.

Now we prove the general case. Let $n=p_{1} \ldots p_{l}$, where $p_{1}, \ldots, p_{l}$ are primes. Prove by induction on $l$. We already proved the base step. Set $n^{\prime}=p_{2} \ldots p_{l}$. Assume we proved the statement for $n^{\prime}$. Then there is $i$ such that $A_{i} \subseteq n^{\prime} \hat{A}$. Set $A^{\prime}=n^{\prime} A$ and $A_{j}^{\prime}=A^{\prime} \cap A_{j}$. It is easy to see that $n^{\prime} \hat{A}=\widehat{A}^{\prime}$. Then there is $j$ such that $A_{j}=\hat{A}_{j}^{\prime} \subseteq p_{1} \widehat{A^{\prime}}=n \hat{A}$.

3.2. Lemmas about abelian groups of finite Prüfer rank. Recall that an abelian group is of finite Prüfer rank if and only if the $\mathbb{Q}$-vector space $A \otimes \mathbb{Q}$ is finite dimensional, the $\mathbb{Z} / p$ vector space ${ }_{p} A$ is finite dimensional for any $p$ and the dimensions are bounded above for all $p$ [15, pp. 33-34]. In other words,

$$
\operatorname{dim}_{\mathbb{Q}}(A \otimes \mathbb{Q})+\max \left\{\operatorname{dim}_{\mathbb{Z} / p}\left({ }_{p} A\right) \mid p \text { is prime }\right\}<\infty .
$$

For for an abelian group $A$ of finite Prüfer rank we set

$$
d_{\mathbb{Q}}(A)=\operatorname{dim}_{\mathbb{Q}}(A \otimes \mathbb{Q}), \quad d_{p}(A)=\operatorname{dim}_{\mathbb{Z} / p}\left({ }_{p} A\right), \quad D_{p}(A)=d_{\mathbb{Q}}(A)+d_{p}(A) .
$$

Lemma 3.4. Let $A$ be an abelian group of finite Prüfer rank. Then $\operatorname{dim}_{\mathbb{Z} / p}(A / p) \leq D_{p}(A)$ and for any subgroup $B \leq A$

$$
D_{p}(B) \leq D_{p}(A), \quad D_{p}(A / B) \leq D_{p}(A) .
$$

Proof. The inequality $D_{p}(B) \leq D_{p}(A)$ is obvious because $d_{\mathbb{Q}}(B) \leq d_{\mathbb{Q}}(A)$ and $d_{p}(B) \leq d_{p}(A)$. We set $E=A / B$ and prove $D_{p}(E) \leq D_{p}(A)$ and $\operatorname{dim}(A / p) \leq D_{p}(A)$.

First we prove it in the case $E \cong(\mathbb{Z} / p)^{n}$. Take a $p$-basic subgroup $A^{\prime}$ of $A$. Then $A^{\prime} / p \cong A / p$, the sequence ${ }_{p} A^{\prime} \gg{ }_{p} A \rightarrow{ }_{p}\left(A / A^{\prime}\right)$ is short exact and $A^{\prime} \cong \mathbb{Z}{ }^{\oplus k} \oplus \mathbb{Z} / p^{m_{1}} \oplus \cdots \oplus \mathbb{Z} / p^{m_{l}}$. Using the monomorphisms $A^{\prime} \otimes \mathbb{Q} \nrightarrow A \otimes \mathbb{Q}$ and ${ }_{p} A^{\prime} \nrightarrow{ }_{p} A$, we obtain $k \leq \operatorname{dim}_{\mathbb{Q}}(A \otimes \mathbb{Q})$ and $l \leq \operatorname{dim}_{\mathbb{Z} / p}\left({ }_{p} A\right)$. Using the isomorphism $A^{\prime} / p \cong A / p$, we get $\operatorname{dim}_{\mathbb{Z} / p}(A / p)=k+l \leq D_{p}(A)$. The epimorphism $A \rightarrow E$ induces an epimorphism $A / p \rightarrow E$. It follows that $D_{p}(E)=n \leq k+l \leq \operatorname{dim}_{\mathbb{Q}}(A \otimes \mathbb{Q})+$ $\operatorname{dim}_{\mathbb{Z} / p}\left({ }_{p} A\right)=D_{p}(A)$.

Now we prove the general case. Denote the preimage of ${ }_{p} E$ in $A$ by $\tilde{A}$. Then we have an epimorphism $\tilde{A} \rightarrow{ }_{p} E$. As we proved above, $\operatorname{dim}_{\mathbb{Z} / p}\left({ }_{p} E\right) \leq \operatorname{dim}_{\mathbb{Q}}(\tilde{A} \otimes \mathbb{Q})+\operatorname{dim}_{\mathbb{Z} / p}\left({ }_{p} \tilde{A}\right)$. Consider 
the diagram with exact rows

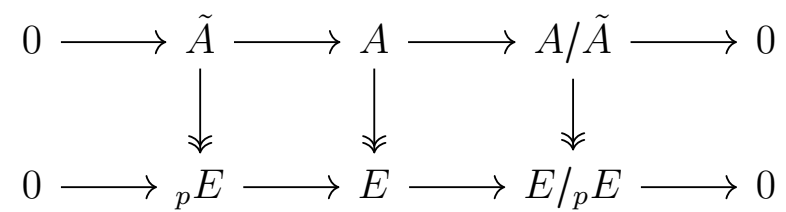

Tensoring by $\mathbb{Q}$ we obtain the following.

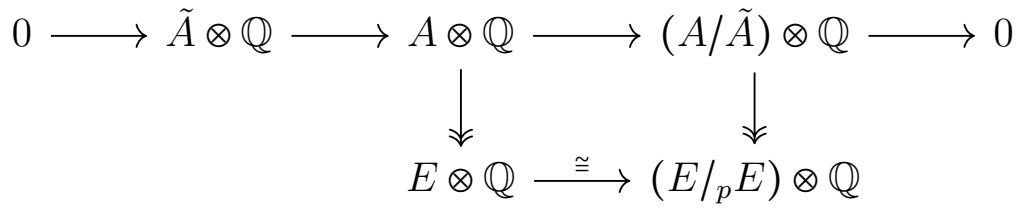

Hence, $\operatorname{dim}_{\mathbb{Q}}(E \otimes \mathbb{Q}) \leq \operatorname{dim}_{\mathbb{Q}}((A / \tilde{A}) \otimes \mathbb{Q})$. Using the two obtained inequalities and the equality $\operatorname{dim}_{\mathbb{Q}}(A \otimes \mathbb{Q})=\operatorname{dim}_{\mathbb{Q}}((A / \tilde{A}) \otimes \mathbb{Q})+\operatorname{dim}_{\mathbb{Q}}(\tilde{A} \otimes \mathbb{Q})$ we get

$$
\begin{gathered}
\operatorname{dim}_{\mathbb{Q}}(E \otimes \mathbb{Q})+\operatorname{dim}_{\mathbb{Z} / p}\left({ }_{p} E\right) \leq \\
\leq \operatorname{dim}_{\mathbb{Q}}((A / \tilde{A}) \otimes \mathbb{Q})+\operatorname{dim}_{\mathbb{Q}}(\tilde{A} \otimes \mathbb{Q})+\operatorname{dim}_{\mathbb{Z} / p}\left({ }_{p} A\right)=\operatorname{dim}_{\mathbb{Q}}(A \otimes \mathbb{Q})+\operatorname{dim}_{\mathbb{Z} / p}\left({ }_{p} A\right) .
\end{gathered}
$$

Lemma 3.5. Let $A$ be an abelian group of finite Prüfer rank, $M$ be a finite $\mathbb{Z} / p[A]$-module and $n \geq 0$. Then

$$
\operatorname{dim}_{\mathbb{Z} / p}\left(H_{n}(A, M)\right) \leq n^{D_{p}(A)-1} \cdot \operatorname{dim}_{\mathbb{Z} / p}(M) .
$$

Proof. We denote by ${ }_{(\text {non- } p)} A$ the sum of $q$-power torsion subgroups for $q \neq p$

$$
\text { (non- } p) A=\bigoplus_{q \neq p}(q) A \text {. }
$$

Any finitely generated subgroup of $A^{\prime} \leq{ }_{(\text {non- } p)} A$ is a finite group of order prime to $p$. Then by Maschke's theorem we obtain $H_{n}\left(A^{\prime}, M\right)=0$ for $n \geq 1$. Since homology commute with direct limits and the group is the direct limit of its finitely generated subgroups, we get $H_{n}\left({ }_{(\text {non- } p)} A, M\right)=0$ for $n \geq 1$. Using the spectral sequence of the short exact sequence $_{(\text {non- } p)} A \rightarrow A \rightarrow A /_{(\text {non- } p)} A$, we obtain $H_{*}(A, M) \cong H_{*}\left(A /_{(\text {non- } p)} A, M_{(\text {non- } p)} A\right)$. Moreover, $D_{p}(A)=D_{p}(A /($ non- $p) A)$. Then we can assume that $A$ is $q$-torsion free for $q \neq p$.

Further in the proof we assume that $A$ is $q$-torsion free for a prime number $q$ distinct from $p$.

Prove for the case where $A$ is one of the cyclic groups $\mathbb{Z}, \mathbb{Z} / p^{k}$. If $A=\mathbb{Z}$, then $H_{1}(A, M)=$ $M^{A} \leq M, H_{n}(A, M)=0$ for $n \geq 2$ and $D_{p}(A)=1$. If $A=\mathbb{Z} / p^{k}=\langle t\rangle$, then, if we set $\mathcal{N}=$ $1+t+t^{2}+\cdots+t^{p^{k}-1} \in \mathbb{Z} / p[A]$, we get $H_{2 m+1}(A, M)=M^{A} / M \cdot \mathcal{N}, H_{2 m}(A, M)=\{m \in M \mid m \cdot \mathcal{N}=$ $0\} / M(1-t)$ and $D_{p}(A)=1$. In all these cases the equation holds.

Now we prove for the case of finitely generated $A$. Then $A=A_{1} \times \cdots \times A_{m}$, where $A_{i}$ is one of the groups $\mathbb{Z}, \mathbb{Z} / p^{k}$. The proof is by induction on $m$. Note that $D_{p}(A)=m$. The base step was proved above. Set $\tilde{A}=A_{2} \times \cdots \times A_{m}$. Consider the spectral sequence $H_{*}\left(A_{1}, H_{*}(\tilde{A}, M)\right) \Rightarrow$ $H_{*}(A, M)$. By induction hypothesis we have $\operatorname{dim}\left(H_{i}(\tilde{A}, M)\right) \leq(n+1)^{m-2} \cdot \operatorname{dim}(M)$ for $i \leq n$. Then $\operatorname{dim}\left(H_{n-i}\left(A_{1}, H_{i}(\tilde{A}, M)\right)\right) \leq(n+1)^{m-2} \cdot \operatorname{dim}(M)$. It follows that $\operatorname{dim}\left(H_{n}(A, M)\right) \leq(n+$ $1) \cdot(n+1)^{m-2} \cdot \operatorname{dim}(M)=(n+1)^{m-1} \cdot \operatorname{dim}(M)$.

Prove the general case. Consider a finitely generated subgroup $A^{\prime} \leq A$. Since $D_{p}\left(A^{\prime}\right) \leq$ $D_{p}(A)$, using that we proved above, we obtain that $\operatorname{dim}\left(H_{n}\left(A^{\prime}, M\right)\right) \leq(n+1)^{D_{p}(A)-1} \cdot \operatorname{dim}(M)$. 
Then the assertion follows from Lemma 2.4, the fact that homology commute with direct filtered limits and that $A$ is the direct limit of its finitely generated subgroups.

Corollary 3.6. Let $A$ be an abelian group of finite Prüfer rank, $M$ be a finite $\mathbb{Z} / p[A]$-module and $n \geq 0$. Then for any subgroup $B \leq A$

$$
\operatorname{dim}_{\mathbb{Z} / p}\left(H_{n}\left(A / B, M_{B}\right)\right) \leq(n+1)^{D_{p}(A)-1} \cdot \operatorname{dim}_{\mathbb{Z} / p}(M) .
$$

In particular, for fixed $A, M, n$ the dimensions of $H_{n}\left(A / B, M_{B}\right)$ are bounded over all subgroups $B \leq A$.

Corollary 3.7. If $A$ is an abelian group such that $A / p$ and ${ }_{p} A$ are finite for any $p$, then $H_{n}(A, \mathbb{Z} / p)$ is finite.

Proof. Take the torsion subgroup $\operatorname{tor}(A)=\bigoplus_{(p)} A$ of $A$. Then ${ }_{(p)} A$ is a an abelian group of finite Prüfer rank. By Lemma $3.5 H_{n}(\operatorname{tor}(A), \mathbb{Z} / p)=H_{n}\left({ }_{(p)} A, \mathbb{Z} / p\right)$ is finite for any $n$. Take the quotient $\operatorname{tf}(A)=A / \operatorname{tor}(A)$. Then $H_{n}(\operatorname{tf}(A), \mathbb{Z} / p)=\Lambda^{n}(\operatorname{tf}(A) / p)$, and hence, it is finite for any $n$. It follows that for any finite $\mathbb{Z} / p$-vector space $H_{n}(\operatorname{tf}(A), V)$ is finite. Consider the spectral sequence of the short exact sequence $\operatorname{tor}(A) \nrightarrow A \rightarrow \operatorname{tf}(A)$. Components of its second page $H_{*}\left(\operatorname{tf}(A), H_{*}(\operatorname{tor}(A), \mathbb{Z} / p)\right)$ are finite. Then $H_{n}(A, \mathbb{Z} / p)$ is finite.

Lemma 3.8. Let $A$ be a abelian group of finite Prüfer rank and $A=A_{1} \supseteq A_{2} \supseteq \ldots$ a sequence of subgroups. Set $\hat{A}=\lim _{\longleftarrow} A / A_{i}$. Then $\hat{A} / p$ and ${ }_{p} \hat{A}$ are finite and

$$
\operatorname{dim}_{\mathbb{Z} / p}(\hat{A} / p) \leq D_{p}(A), \quad \operatorname{dim}_{\mathbb{Z} / p}\left({ }_{p} \hat{A}\right) \leq D_{p}(A) .
$$

Proof. Set $B_{i}=A / A_{i}$. Lemma 3.2 implies that $\hat{A} / p=\lim _{\longleftarrow}\left(B_{i} / p\right)$ and ${ }_{p} \hat{A}=\lim _{\longleftarrow}\left({ }_{p} B_{i}\right)$. By Lemma

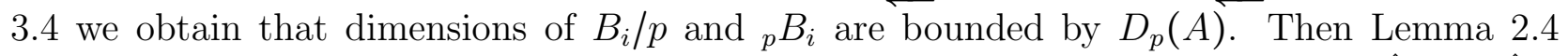
implies that the sequences ${ }_{p} B_{i}$ and $B_{i} / p$ are quasistable and the dimensions of ${ }_{p} \hat{A}$ and $\hat{A} / p$ are bounded by $D_{p}(A)$.

\subsection{Completions of abelian and solvable groups of finite Prüfer rank.}

Proposition 3.9. Let $A$ be an abelian group such that $A / p$ and ${ }_{p} A$ are finite for any $p$ and $A=A_{1} \supseteq A_{2} \supseteq \ldots$ be a sequence of subgroups and $M$ be an $\mathbb{Z} / p[A]$-module. Assume that

(1) the groups ${ }_{p}\left(A / A_{i}\right)$ and $A_{i} / p$ are finite for any $i$;

(2) for any $i$ there $i s ~ j>i$ such that $A_{j} \subseteq p A_{i}$;

(3) ${ }_{p} A_{i}=0$ for big enough $i$;

(4) $A_{i}$ acts trivially on $M$ for big enough $i$.

Then the obvious morphisms are isomorphisms

$$
H_{*}(A, M) \cong \lim _{\longleftarrow} H_{*}\left(A / A_{i}, M_{A_{i}}\right), \quad H^{*}(A, M) \cong \lim _{\longrightarrow} H^{*}\left(A / A_{i}, M^{A_{i}}\right) .
$$

Proof. Since $A_{i}$ acts trivially on $M$ for big enough $i$, we have $H_{n}\left(A_{i}, M\right) \cong H_{n}\left(A_{i}, \mathbb{Z} / p\right) \otimes M$ for big enough $i$. By assumption for any fixed $i$ there exist $j>i$ such that $A_{j} \subseteq p A_{i}$ and ${ }_{p} A_{j}=0$. Then by Lemma 3.1 we obtain that for any $n$ and big enough $i$ (so big that $M$ is a trivial $A_{i}$-module) there exists $j>i$ such that the map $H_{n}\left(A_{j}, M\right) \rightarrow H_{n}\left(A_{i}, M\right)$ is trivial. Therefore, the sequence $H_{n}\left(A_{i}, M\right)$ is zero-equivalent for any $n$. For any $i$ we have a spectral sequence $H_{*}\left(A / A_{i}, H_{*}\left(A_{i}, M\right)\right) \Rightarrow H_{*}(A, M)$. Since ${ }_{p}\left(A / A_{i}\right), A_{i} / p$ are finite, we have that the second page consists of finite groups (Corollary [3.7). The functor of inverse limit is exact on the category of finite abilian groups, and hence, we get the spectral sequence $\lim _{\longleftarrow} H_{*}\left(A / A_{i}, H_{*}\left(A_{i}, M\right)\right) \Rightarrow H_{*}(A, M)$. Since the inverse sequence 
$H_{n}\left(A_{i}, M\right)$ is zero-equivalent, we obtain $\lim _{\longleftarrow} H_{*}\left(A / A_{i}, H_{n}\left(A_{i}, M\right)\right)=0$ for $n \geq 1$. Then $\lim _{\leftarrow} H_{*}\left(A / A_{i}, M\right) \cong H_{*}(A, M)$.

For cohomology the proof is similar, if we use the formula $H^{*}(A, M) \cong H_{*}\left(A, M^{\vee}\right)^{\vee}$ for a group $A$ and a finite $\mathbb{Z} / p[A]$-module $M$.

Proposition 3.10. Let $A$ be an abelian group of finite Prüfer rank and let $A \supseteq A_{1} \supseteq A_{2} \supseteq \ldots$ be a sequence of subgroups. Set $\hat{A}=\lim A / A_{i}$ and $\hat{A}_{i}=\operatorname{Ker}\left(\hat{A} \rightarrow A / A_{i}\right)$. Assume that $M$ is a finite $\mathbb{Z} / p[\hat{A}]$-module. Then $\hat{A}_{i}$ acts trivially on $M$ for big enough $i$, the dimensions of the sequences $H_{n}\left(A / A_{i}, M_{\hat{A}_{i}}\right)$ and $H^{n}\left(A / A_{i}, M^{\hat{A}_{i}}\right)$ are bonded for any $n$, the groups $H_{n}(\hat{A}, M)$ and $H^{n}(\hat{A}, M)$ are finite for any $n$, and the obvious maps are isomorphisms

$$
\begin{gathered}
H_{*}(\hat{A}, M) \cong \lim _{\longleftarrow} H_{*}\left(A / A_{i}, M_{\hat{A}_{i}}\right), \\
H^{*}(\hat{A}, M) \cong \lim _{\longrightarrow} H^{*}\left(A / A_{i}, M^{\hat{A}_{i}}\right) \cong H_{u}^{*}(\hat{A}, M) .
\end{gathered}
$$

In particular, $M$ is a cohomologically discrete $\hat{A}$-module.

Proof. It is easy to see that $\hat{A}_{k}=\lim _{i>k} A_{k} / A_{i}$, that $\hat{A} / \hat{A}_{k} \cong A / A_{k}$ are of finite Prüfer rank and $\cap \hat{A}_{i}=0$. In particular, ${ }_{p} \hat{A}_{i}=0$ for big enough $i$. By Lemma 3.8 we obtain that $\hat{A}_{k} / p$ and ${ }_{p}\left(\hat{A}_{i}\right)$ are finite. By Lemma 3.3 we obtain that for any $i$ there is $j>i$ such that $\hat{A}_{j} \subseteq p \hat{A}_{i}$. Since $M$ is finite, $\mathrm{G}=\operatorname{Aut}_{\mathbb{Z}}(M)$ is finite. The action of $\hat{A}$ on $M$ is given by a homomorphism $\hat{A} \rightarrow \mathrm{G}$. Since $|\mathrm{G}| \cdot \hat{A}$ is in the kernel of the homomorphism, $\hat{A}_{i}$ acts trivially on $M$ for big $i$ (Lemma 3.3). Then we can use Proposition 3.9 for the sequence $\hat{A} \supseteq \hat{A}_{1} \supseteq \hat{A}_{2} \ldots$ and obtain the isomorphisms $H_{*}(\hat{A}, M) \cong \lim H_{*}\left(A / A_{i}, M_{\hat{A}_{i}}\right)$ and $H^{*}(\hat{A}, M) \cong \lim _{\longrightarrow} H^{*}\left(A / A_{i}, M^{\hat{A}_{i}}\right)$. Moreover, we obtain $M=M_{\hat{A}_{i}}=M^{\widehat{A_{i}}}$ for big enough $i$. By Corollary [3.6] we obtain that the dimensions of $H_{n}\left(A / A_{i}, M_{\hat{A}_{i}}\right)$ and $H^{n}\left(A / A_{i}, M^{\hat{A}_{i}}\right)$ are bounded for a fixed $n$. Then Lemma 2.4 implies that these sequences are quasiconstant. The last isomorphism follows from Proposition 2.6 .

Note that solvable group $G$ is of finite Prüfer rank if and only if there is a finite sequence of normal subgroups $G=U_{1} \supseteq U_{2} \supseteq \cdots \supseteq U_{s}=1$ such that $U_{i} / U_{i+1}$ are abelian groups of finite Prüfer rank.

Theorem 3.11. Let $G$ be a solvable group group of finite Prüfer rank and let $G \supseteq G_{1} \supseteq G_{2} \supseteq \ldots$ be a sequence of normal subgroups. Set $\hat{G}=\lim G / G_{i}$ and $\hat{G}_{i}=\operatorname{Ker}\left(\hat{G} \rightarrow G / G_{i}\right)$. Assume that $M$ is a finite $\mathbb{Z} / p[\hat{G}]$-module. Then $\hat{G}_{i}$ acts trivially on $M$ for big enough $i$, the dimensions of the sequences $H_{n}\left(G / G_{i}, M_{\hat{G}_{i}}\right)$ and $H^{n}\left(G / G_{i}, M^{\hat{G}_{i}}\right)$ are bounded for any $n$, the groups $H_{n}(\hat{G}, M)$ and $H^{n}(\hat{G}, M)$ are finite for any $n$, and the obvious maps are isomorphisms

$$
\begin{gathered}
H_{*}(\hat{G}, M) \cong \lim _{\longleftarrow} H_{*}\left(G / G_{i}, M_{\hat{G}_{i}}\right), \\
H^{*}(\hat{G}, M) \cong \lim _{\longrightarrow} H^{*}\left(G / G_{i}, M^{\hat{A}_{i}}\right) \cong H_{u}^{*}(\hat{G}, M) .
\end{gathered}
$$

In particular, $M$ is a cohomologically discrete $\hat{G}$-module.

Proof. Let $G=U_{1} \supseteq U_{2} \supseteq \cdots \supseteq U_{s}=1$ be a finite sequence of normal subgroups such that $U_{i} / U_{i+1}$ are abelian groups of finite Prüfer rank and $M$ be a finite $\mathbb{Z} / p[\hat{G}]$-module. The proof is by induction on $s$. If $s=1$, it follows from Proposition 3.10. Prove the induction step. Set $A=U_{s-1}, A_{i}=A \cap G_{i}, \hat{A}=\lim _{\longleftarrow} A / A_{i}, G^{\prime}=G / A, G_{i}^{\prime}=G_{i} A / A, \quad \hat{G}^{\prime}=\lim G^{\prime} / G_{i}^{\prime}$. Then there are 
short exact sequences $\hat{A} \nrightarrow \hat{G} \rightarrow \hat{G}^{\prime}$. Note that $G^{\prime} / G_{i}^{\prime}=G / G_{i} A$. It follows that there is a short exact sequence $A / A_{i} \nrightarrow G / G_{i} \rightarrow G^{\prime} / G_{i}^{\prime}$. We set $\hat{A}_{i}=\operatorname{Ker}\left(\hat{A} \rightarrow A / A_{i}\right), \hat{G}_{i}=\operatorname{Ker}\left(\hat{G} \rightarrow G / G_{i}\right)$ and $\hat{G}_{i}^{\prime}=\operatorname{Ker}\left(\hat{G}^{\prime} \rightarrow G^{\prime} / G_{i}^{\prime}\right)$. Then we obtain a commutative diagram, whose columns are short exact sequences and the second and third rows are short exact sequences.

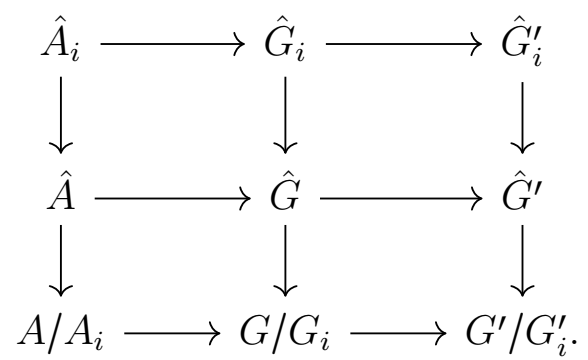

Using $3 \times 3$-lemma, we obtain that $\hat{A}_{i} \nrightarrow \hat{G}_{i} \rightarrow \hat{G}_{i}^{\prime}$ is a short exact sequence. By Proposition 3.10 $\hat{A}_{i}$ acts trivially on $M$ for big enough $i$. It follows that the action of $\hat{G}_{i}$ on $M$ factors through $\hat{G}_{i}^{\prime}$ for big enough $i$. Then using the induction hypothesis, we obtain $\hat{G}_{j}^{\prime}$ acts trivially on $M$ for big enough $j$. Therefore, $\hat{G}_{j}$ acts trivially on $M$ for big enough $j$. By Proposition 3.10 we obtain that there is an isomorphism $H^{\star}(\hat{A}, M) \cong \lim _{\longrightarrow} H^{*}\left(A / A_{i}, M^{\hat{A}_{i}}\right)$, all these groups are finite $\mathbb{Z} / p\left[\hat{G}^{\prime}\right]$-modules, where $\hat{G}^{\prime}=\lim G / G_{i} A$, and the sequences $H^{*}\left(A / A_{i}, M^{\hat{A}_{i}}\right)$ have bounded dimensions, and hence, they are quasiconstant. Then by induction hypothesis we have $H^{*}\left(\hat{G}^{\prime}, H^{*}(\hat{A}, M)\right) \cong H_{u}^{*}\left(\hat{G}^{\prime}, H^{*}(\hat{A}, M)\right)$. By Proposition 2.6 we have $H_{u}^{*}\left(\hat{G}^{\prime}, H^{*}(\hat{A}, M)\right) \cong$ $\lim _{\longrightarrow} H^{*}\left(G^{\prime} / G_{i}^{\prime}, H^{*}\left(A / A_{i}, M\right)\right)$. It follows that $H^{*}(\hat{G}, M) \cong \lim _{\longrightarrow} H^{*}\left(G / G_{i}, M^{\hat{G}_{i}}\right)$. Proposition 2.6 implies that $\lim _{\longrightarrow} H^{*}\left(G / G_{i}, M^{\hat{G}_{i}}\right) \cong H_{u}^{*}(\hat{G}, M)$. The proof for homology is similar.

\section{R-COMPLETIONS}

4.1. Definitions. Let $R \in\{\mathbb{Z}, \mathbb{Z} / p, \mathbb{Q}\}$. Following Bousfield [7] we define lower $R$-central series of a group $G$ by recursion

$$
\gamma_{i+1}^{R} G=\operatorname{Ker}\left(\gamma_{i}^{R} G \rightarrow \gamma_{i}^{R} G /\left[\gamma_{i}^{R} G, G\right] \otimes R\right),
$$

where $\gamma_{1}^{R} G=G$. Then $R$-completion of $G$ is defined as follows

$$
\hat{G}_{R}= \begin{cases}\lim _{\leftarrow} G / \gamma_{i}^{R} G, & \text { if } R \in\{\mathbb{Z}, \mathbb{Z} / p\} \\ \lim _{\longleftarrow}\left(G / \gamma_{i}^{\mathbb{Q}} G \otimes \mathbb{Q}\right), & \text { if } R=\mathbb{Q},\end{cases}
$$

where $-\otimes \mathbb{Q}$ is Malcev completion functor.

Remark 4.1. It is easy to prove by induction that

$$
\gamma_{i}^{\mathbb{Q}} G=\sqrt{\gamma_{i}^{\mathbb{Z}} G}:=\left\{g \in G \mid g^{n} \in \gamma_{i}^{\mathbb{Z}} G \text { for some } n\right\} .
$$

It follows that $G / \gamma_{i}^{\mathbb{Q}} G \otimes \mathbb{Q}=G / \gamma_{i}^{\mathbb{Z}} G \otimes \mathbb{Q}$. Then $\hat{G}_{\mathbb{Q}}=\lim _{\longleftarrow}\left(G / \gamma_{i}^{\mathbb{Z}} G \otimes \mathbb{Q}\right)$.

A group $G$ is said to be $H R$-good if the map $H_{2}(G, R) \rightarrow H_{2}\left(\hat{G}_{R}, R\right)$ is an epimorphism. Moreover, it is interesting when the map $H_{2}(G, \mathbb{Z} / p) \rightarrow H_{2}\left(\hat{G}_{\mathbb{Z}}, \mathbb{Z} / p\right)$ is an epimorphism.

$G$ is called $R$-prenilpotent if $\gamma_{i}^{R} G=\gamma_{i+1}^{R} G$ for big enough $i$. In this case $\hat{G}_{R}=G / \gamma_{i}^{R} G$ for $R \in\{\mathbb{Z}, \mathbb{Z} / p\}$ and $\hat{G}_{\mathbb{Q}}=G / \gamma_{i}^{\mathbb{Q}} \otimes \mathbb{Q}$ for big enough $i$. It follows that $\hat{G}_{R}$ is discrete, and in particular, cohomomologically discrete over any ring. 
4.2. 2-cohomologicaly discrete $R$-completions. We are interested in the map

$$
H_{n}(G, K) \longrightarrow H_{n}\left(\hat{G}_{R}, K\right) .
$$

We denote by $\Phi_{\alpha}^{R} H_{n}(G, K) \alpha$ th term of Dwyer filtration, which is defined as

$$
\Phi_{\alpha}^{R} H_{n}(G, K)=\operatorname{Ker}\left(H_{n}(G, K) \rightarrow H_{n}\left(G / \gamma_{\alpha}^{R} G, K\right)\right) .
$$

Using that $H_{n}\left(G / \gamma_{i}^{\mathbb{Q}} G, \mathbb{Q}\right)=H_{n}\left(G / \gamma_{i}^{\mathbb{Q}} G \otimes \mathbb{Q}, \mathbb{Q}\right.$ ) (Proposition 2.5 of [12]) we obtain that the map (4.1) factors through $H_{n}\left(G / \gamma_{i}^{R} G, K\right)$ for any finite $i$. On the other hand, the map $G \rightarrow \hat{G}_{R}$ factors through $G / \gamma_{\omega} G$. Hence

$$
\Phi_{\omega}^{R} H_{n}(G, K) \subseteq \operatorname{Ker}\left(H_{n}(G, K) \rightarrow H_{n}\left(\hat{G}_{R}, K\right)\right) \subseteq \bigcap_{i=1}^{\infty} \Phi_{i}^{R} H_{n}(G, K) .
$$

Lemma 4.2. Let $G$ be a group and $S$ be a quotient of $R$. Set $\Phi_{i}:=\Phi_{i}^{R} H_{2}(G, S)$.

(1) Then $\lim _{\longleftarrow} H_{2}(G, S) / \Phi_{i}=\lim _{\longleftarrow} H_{2}\left(G / \gamma_{i}^{R} G, S\right)$ and there is an exact sequence

$$
0 \rightarrow \bigcap_{i=1}^{\infty} \Phi_{i} \rightarrow H_{2}(G, S) \rightarrow \lim _{\longleftarrow} H_{2}\left(G / \gamma_{i}^{R} G, S\right) \rightarrow \lim ^{1} \Phi_{i} \rightarrow 0 .
$$

(2) Moreover, if $S=K$ and $H_{2}(G, K)$ is finite dimensional, then there are short exact sequences

$$
0 \longrightarrow \Phi_{j} \longrightarrow H_{2}(G, K) \longrightarrow \lim _{\longleftarrow} H_{2}\left(G / \gamma_{i}^{R} G, K\right) \longrightarrow 0
$$

for big enough $j$, and the inverse sequence $H_{2}\left(G / \gamma_{i}^{R} G, K\right)$ is quasiconstant.

Proof. The 5-term exact sequence gives the exact sequence

$$
0 \longrightarrow H_{2}(G, S) / \Phi_{i} \longrightarrow H_{2}\left(G / \gamma_{i}^{R} G, S\right) \longrightarrow \gamma_{i}^{R} G / \gamma_{i}^{R} G \otimes S \longrightarrow 0 .
$$

Since $\gamma_{i}^{R} G / \gamma_{i}^{R} G \otimes S$ is zero-equivalent, $\lim _{\longleftarrow} H_{2}(G, S) / \Phi_{i}=\lim _{\breve{G}} H_{2}\left(G / \gamma_{i}^{R} G, S\right)$. Then the (1) follows from the short exact sequence $\Phi_{i} \hookrightarrow H_{2}(G, S) \rightarrow H_{2}(\overleftarrow{G, S}) / \Phi_{i}$

If $S=K$ and $H_{2}(G, K)$ is finite dimensional, then $V_{i}$ and $\Phi_{i}$ stabilises. Hence $\lim ^{1} \Phi_{i}=0$ and (4.3) implies that $H_{2}\left(G / \gamma_{i}^{R} G, K\right)$ is quasiconstant.

Proposition 4.3. Let $G$ be a group such that $H_{1}(G, R)$ is finitely generated over $R$ and $H_{2}(G, K)$ is finite dimensional. Set $\Phi_{i}:=\Phi_{i}^{R} H_{2}(G, K)$. Then the following are equivalent.

(1) $\hat{G}_{R}$ is 2-cohomologically discrete over $K$.

(2) The obvious maps give a short exact sequence

$$
0 \longrightarrow \Phi_{i} \longrightarrow H_{2}(G, K) \longrightarrow H_{2}\left(\hat{G}_{R}, K\right) \longrightarrow 0
$$

for big enough $i$.

(3) $H_{2}\left(\hat{G}_{R}, K\right) \cong \lim _{\longleftarrow} H_{2}\left(G / \gamma_{i}^{R} G, K\right)$.

Proof. Since $H_{1}(G, R)$ is finitely generated over $R$, we have $H_{1}(G, R) \cong H_{1}\left(\hat{G}_{R}, R\right)$ (see [7]). Thus $H^{1}(G, K)=H^{1}\left(G / \gamma_{i}^{R} G, K\right)=H^{1}\left(\hat{G}_{R}, K\right)$ for any $i>1$. Hence we need to think only about the second (co)homology.

Lemma4.2 implies that (2) and (3) are equivalent. Moreover, it implies that $H_{2}\left(G / \gamma_{i}^{R} G, K\right)$ is quasiconstant and its inverse limit is finite dimensional. By Lemma 2.3 we get that $H^{2}\left(G / \gamma_{i}^{R} G, K\right)$ in a quasiconstant direct sequence with a finite dimensional inverse limit. It follows that $\left(\lim _{\longleftarrow} H_{2}\left(G / \gamma_{i}^{R} G, K\right)\right)^{*}=\lim _{\longrightarrow} H^{2}\left(G / \gamma_{i}^{R} G, K\right) \cong H_{u}^{2}\left(\hat{G}_{R}, K\right)$ and $\left(\stackrel{\lim }{\longrightarrow} H^{2}\left(G / \gamma_{i}^{R} G, K\right)\right)^{*}=\lim _{\longleftarrow} H_{2}\left(G / \gamma_{i}^{R} G, K\right)$, where $\left(-\overrightarrow{)^{*}}=\operatorname{Hom}_{K}(-, K)\right.$. Using this, we get that $(3)$ is equivalent to $\overleftarrow{(1)}$ 
Remark 4.4. Using Proposition 4.3, the main result of [13] can be reformulated as follows. If $G$ is a finitely presented metabelian group, then $\hat{G}_{R}$ is 2-cohomologically discrete over $K$.

4.3. $\mathbb{Q}$-prenilpotent groups. Note that by definition $G / \gamma_{i}^{\mathbb{Q}} G$ is torsion free.

Lemma 4.5. A group $G$ is $\mathbb{Q}$-prenilpotent if and only if the sequence $G / \gamma_{i}^{\mathbb{Q}} G \otimes \mathbb{Q}$ stabilises.

Proof. If $G$ is $\mathbb{Q}$-prenilpotent, then obviously the sequence $G / \gamma_{i}^{\mathbb{Q}} G \otimes \mathbb{Q}$ stabilises. Assume now that $G / \gamma_{i}^{\mathbb{Q}} G \otimes \mathbb{Q}$ stabilises and prove that $G$ is $\mathbb{Q}$-prenilpotent. Since $-\otimes \mathbb{Q}$ is an exact functor, this means that $\gamma_{i}^{\mathbb{Q}} G / \gamma_{i+1}^{\mathbb{Q}} G \otimes \mathbb{Q}=0$ for big enough $i$. Using that $\gamma_{i}^{\mathbb{Q}} G / \gamma_{i+1}^{\mathbb{Q}} G$ is torsion free, we obtain that $\gamma_{i}^{\mathbb{Q}} G / \gamma_{i+1}^{\mathbb{Q}} G=0$ for big enough $i$. Thus $G$ is $\mathbb{Q}$-prenilpotent.

Lemma 4.6. Let $A \nrightarrow G \rightarrow G^{\prime \prime}$ be a short exact sequence of groups such that

(1) $G^{\prime \prime}$ is $\mathbb{Q}$-prenilpotent.

(2) $A$ is an abelian group such that $A \otimes \mathbb{Q}$ is finite dimensional.

Then $G$ is $\mathbb{Q}$-prenilpotent.

Proof. We assue that $A$ is a normal subgroup in $G$ and $A \nrightarrow G$ is the embedding. Set $V_{i}=$ $\left(A / A \cap \gamma_{i}^{\mathbb{Q}} G\right) \otimes \mathbb{Q}$. Then $V_{i}$ is a quotient of $A \otimes \mathbb{Q}$. Consider the short exact sequence

$$
0 \longrightarrow V_{i} \longrightarrow G / \gamma_{i}^{\mathbb{Q}} G \otimes \mathbb{Q} \longrightarrow G^{\prime \prime} / \gamma_{i}^{\mathbb{Q}} G^{\prime \prime} \otimes \mathbb{Q} \longrightarrow 1
$$

Using that $G^{\prime \prime}$ is $\mathbb{Q}$-prenilpotent and $A \otimes \mathbb{Q}$ is finitedimensional, we get that $G^{\prime \prime} / \gamma_{i}^{\mathbb{Q}} G^{\prime \prime} \otimes \mathbb{Q}$ and $V_{i}$ stabilise. Then $G / \gamma_{i}^{\mathbb{Q}} G \otimes \mathbb{Q}$ stabilises. Then by Lemma 4.5 we obtain that $G$ is $\mathbb{Q}$-prenilpotent.

Proposition 4.7. Let $G$ be a solvable group with a finite sequence of normal subgroups $G=$ $U_{1} \supseteq U_{2} \supseteq \cdots \supseteq U_{s}=1$ such that $U_{i} / U_{i+1}$ are abelian and $U_{i} / U_{i+1} \otimes \mathbb{Q}$ are finite dimensional. Then $G$ is $\mathbb{Q}$-prenilpotent. In particular, $\hat{G}_{\mathbb{Q}}$ is cohomologically discrete over $\mathbb{Q}$. Moreover, if $G$ is finitely generated, then it is $H \mathbb{Q}$-good.

Proof. Using the Lyndon-Hochschild-Serre spectral sequence and induction on $s$, it is easy to see that $H_{2}(G, \mathbb{Q})$ is finite dimensional. Then the assertion follows from Lemma 4.6 by induction and Proposition 4.3.

Remark 4.8. Note that we can not replace $\mathbb{Q}$ by $\mathbb{Z}$ or $\mathbb{Z} / p$ in Proposition 4.7. For example, consider the group $G=\mathbb{Z} \rtimes \mathbb{Z} / 2$ with the sign action of $\mathbb{Z} / 2$ on $\mathbb{Z}$. Then we have a short exact sequence $\mathbb{Z} \rightarrow G \rightarrow \mathbb{Z} / 2$, where $\mathbb{Z} / 2 \otimes R$ and $\mathbb{Z} \otimes R$ are finitely generated over $R$ for $R=\mathbb{Z}, \mathbb{Z} / 2$. But $G$ is not $R$-prenilpotent for $R=\mathbb{Z}, \mathbb{Z} / 2$ because $\gamma_{i}^{\mathbb{Z}}(G)=\gamma_{i}^{\mathbb{Z} / 2}(G)=2^{i-1} \mathbb{Z} \rtimes 0$ for $i \geq 2$.

4.4. Completions of solvable groups of finite Prüfer rank. Recall that a solvable group $G$ is of finite Prüfer rank if there is a finite sequence of normal subgroups $G=U_{1} \supseteq \cdots \supseteq U_{s}=1$ such that for any $i$ the group $U_{i} / U_{i+1}$ is an abelian group of finite Prüfer rank.

Theorem 4.9. Let $G$ be a solvable group of finite Prüfer rank. Then $\hat{G}_{\mathbb{Z}}$ and $\hat{G}_{\mathbb{Z} / p}$ are cohomologically discrete over $\mathbb{Z} / p$. Moreover, if $G$ is finitely generated, then $H_{2}(G, \mathbb{Z} / p) \rightarrow$ $H_{2}\left(\hat{G}_{R}, \mathbb{Z} / p\right)$ is an epimorphism for $R \in\{\mathbb{Z}, \mathbb{Z} / p\}$.

Proof. Cohomological discreteness follows from Theorem 3.11. Using that $H_{n}(A, \mathbb{Z} / p)$ is finite for an abelian group $A$ of finite Prüfer rank, by induction, it is easy to check that $H_{n}(G, \mathbb{Z} / p)$ is finite. Then the epimorphism follows from Proposition 4.3 . 
Proposition 4.10. Let $A$ be a abelian group such that $A / p$ and ${ }_{p} A$ are finite. Then $\hat{A}_{\mathbb{Z} / p}$ is a finitely generated $\mathbb{Z}_{p}$-module. Moreover, if $\operatorname{tor}(A)$ is finite and $M$ is a finite $\mathbb{Z} / p\left[\hat{A}_{\mathbb{Z} / p}\right]$-module, then

$$
H_{*}(A, M)=H_{*}\left(\hat{A}_{\mathbb{Z} / p}, M\right) .
$$

Proof. The multiplication by $p^{i}$ gives an epimorphism $A / p \rightarrow p^{i} A / p^{i+1} A$. It follows that $p^{i} A / p^{i+1} A$ is finite and $A / p^{i}$ is finite for any $i$. Then $\hat{A}_{\mathbb{Z} / p}$ is a profinite abelian group. Note that $A / p^{i}$ is a product of cyclic $p$-groups, and the number of the factors does not depend on $i$ because $\left(A / p^{i}\right) / p=A / p$. It follows that $\hat{A}_{\mathbb{Z} / p}$ has a finite set of generators converging to 1 (see Lemma 2.5.3 of [16]). For any other prime number $q \neq p$ we have $\left(\hat{A}_{\mathbb{Z} / p}\right) / q=\lim _{\longleftarrow}\left(A / p^{i}\right) / q=0$ (Lemma 3.2). Then by Theorem 4.3.5 of [16] we obtain $\hat{A}_{\mathbb{Z} / p}$ is a finitely generated $\mathbb{Z}_{p}$-module.

Assume that $\operatorname{tor}(A)$ is finite. Hence ${ }_{p}\left(p^{i} A\right)=0$ for big enough $i$. Note that $p^{i} A / p^{i+1} A$ is finite and $A / p^{i}$ is finite. Consider the group of automorphisms $G=\operatorname{Aut}_{\mathbb{Z}}(M)$ and assume that $p^{t}$ is the largest power of $p$ that divides $|G|$. Hence $p^{t} \hat{A}_{\mathbb{Z} / p} \subseteq \operatorname{Ker}\left(\hat{A}_{\mathbb{Z} / p} \rightarrow G\right)$. It follows that $p^{t} \hat{A}_{\mathbb{Z} / p}$ acts trivially on $M$. Then we can use Proposition 3.9 both for $A$ and $\hat{A}_{\mathbb{Z} / p}$. The assertion follows.

Remark 4.11. If $A$ is an abelian group of finite Prüfer rank but the torsion subgroup is not finite, the isomorphism (4.4) fails. For example, if $\mathbb{Z} / p^{\infty}=\underset{\lim }{\longrightarrow} \mathbb{Z} / p^{i}$, then $\left(\mathbb{Z} / p^{\infty}\right)_{\mathbb{Z} / p}^{\wedge}=0$ and

$$
\mathbb{Z} / p=H_{2}\left(\mathbb{Z} / p^{\infty}, \mathbb{Z} / p\right) \neq H_{2}\left(\left(\mathbb{Z} / p^{\infty}\right)_{\mathbb{Z} / p}^{\wedge}, \mathbb{Z} / p\right)=0 .
$$

\section{Metabelian groups over $C$}

5.1. Completion of modules. Let $\Lambda$ be a Noetherian commutative ring and $\mathfrak{a} \triangleleft \Lambda$ be an ideal. The $\mathfrak{a}$-adic completion of a $\Lambda$-module $M$ is the inverse limit $\hat{M}_{\mathfrak{a}}=\underset{\lim }{\overleftarrow{\Lambda}} M / M \mathfrak{a}^{i}$. Denote by $\varphi_{M}: M \rightarrow \hat{M}_{\mathfrak{a}}$ the obvious map. The completion of the ring itself $\overleftarrow{\Lambda}_{\mathfrak{a}}$ is a Noetherian commutative ring [5, III, $\S 3$, Cor. of Prop.8] and $\hat{M}_{\mathfrak{a}}$ has a natural structure of $\hat{\Lambda}_{\mathfrak{a}}$-module. Moreover,

$$
\hat{M}_{\mathfrak{a}}=\varphi_{M}(M) \cdot \hat{\Lambda}_{\mathfrak{a}}
$$

[5, III, $\S 3$, Cor.1]. The functor $M \mapsto \hat{M}_{\mathfrak{a}}$ is exact on the category of finitely generated $\Lambda$ modules, there is an isomorphism

$$
\hat{M}_{\mathfrak{a}} \cong M \otimes_{\Lambda} \hat{\Lambda}_{\mathfrak{a}}
$$

for a finitely generated module $M$ [5, III, §3, Th.3]. In particular, if $N \leq M$, we can identify $\hat{N}_{\mathfrak{a}}$ with the submodule in $\hat{M}_{\mathfrak{a}}$.

Consider the ring homomorphism $\varphi=\varphi_{\Lambda}: \Lambda \rightarrow \hat{\Lambda}_{\mathfrak{a}}$. Denote by $\hat{\mathfrak{a}}=\operatorname{Ker}\left(\hat{\Lambda}_{\mathfrak{a}} \rightarrow \Lambda / \mathfrak{a}\right)$. Applying the functor of the $\mathfrak{a}$-adic completion to the short exact sequence $\mathfrak{a} \rightarrow \Lambda \rightarrow \Lambda / \mathfrak{a}$ we get $\hat{\mathfrak{a}}=\hat{\mathfrak{a}}_{\mathfrak{a}}$. Using (5.1) we obtain $\hat{\mathfrak{a}}=\hat{\Lambda}_{\mathfrak{a}} \cdot \varphi(\mathfrak{a})$. If we identify $\widehat{\left(\mathfrak{a}^{i}\right)} \mathfrak{a}$ with the ideal of $\hat{\Lambda}_{\mathfrak{a}}$ and use (5.1), we obtain

$$
\widehat{\left(\mathfrak{a}^{i}\right)_{\mathfrak{a}}}=\varphi_{\mathfrak{a}^{i}}\left(\mathfrak{a}^{i}\right) \cdot \hat{\Lambda}_{\mathfrak{a}}=\hat{\Lambda}_{\mathfrak{a}} \cdot \varphi\left(\mathfrak{a}^{i}\right)=\hat{\mathfrak{a}}^{i} .
$$

Tensoring the short exact sequence $\hat{\mathfrak{a}}^{i} \rightarrow \hat{\Lambda}_{\mathfrak{a}} \rightarrow \Lambda / \mathfrak{a}^{i}$ on $M$, we obtain the exact sequence $M \otimes_{\Lambda} \hat{\mathfrak{a}}^{i} \rightarrow \hat{M} \rightarrow M / M \mathfrak{a}^{i} \rightarrow 0$, which implies

$$
M / M \mathfrak{a}^{i}=\hat{M}_{\mathfrak{a}} / \hat{M}_{\mathfrak{a}} \hat{\mathfrak{a}}^{i} .
$$


In particular, $\Lambda / \mathfrak{a}^{i}=\hat{\Lambda}_{\mathfrak{a}} / \hat{\mathfrak{a}}^{i}$. It follows that $\left(\hat{\Lambda}_{\mathfrak{a}}\right)_{\hat{\mathfrak{a}}}=\hat{\Lambda}_{\mathfrak{a}}$. Assume that $\mathcal{M}$ is a finitely generated $\hat{\Lambda}_{\mathfrak{a}}$-module. Using (5.3) we obtain $\mathcal{M} \cdot \hat{\mathfrak{a}}^{i}=\mathcal{M} \cdot \mathfrak{a}^{i}$. Then, using (5.2) , the isomorphism $\left(\hat{\Lambda}_{\mathfrak{a}}\right)_{\hat{\mathfrak{a}}}=\hat{\Lambda}_{\mathfrak{a}}$ and the equality $\mathcal{M} \cdot \hat{\mathfrak{a}}^{i}=\mathcal{M} \cdot \mathfrak{a}^{i}$, we get

$$
\hat{\mathcal{M}}_{\hat{\mathfrak{a}}}=\hat{\mathcal{M}}_{\mathfrak{a}}=\mathcal{M}
$$

Lemma 5.1. Let $\Lambda$ be a Noetherian commutative ring, $\mathfrak{a}, \mathfrak{b}$ are ideals of $\Lambda$ and $M$ be a finitely generated $\Lambda$-module. Then

$$
\left(\hat{M}_{\mathfrak{a}}\right)_{\mathfrak{b}}^{\wedge}=\hat{M}_{\mathfrak{a}+\mathfrak{b}}=\left(\hat{M}_{\mathfrak{b}}\right)_{\mathfrak{a}}^{\wedge}
$$

Proof. Note that $\hat{M} \mathfrak{a}^{i}=\hat{M} \hat{\mathfrak{a}}^{i}$. Then we have $\hat{M}_{\mathfrak{a}} / \hat{M}_{\mathfrak{a}} \mathfrak{a}^{i}=M / M \mathfrak{a}^{i}$. It follows that

$$
\frac{M}{M \mathfrak{a}^{i}+M \mathfrak{b}^{j}}=\frac{\hat{M}_{\mathfrak{a}}}{\hat{M}_{\mathfrak{a}} \mathfrak{a}^{i}+\hat{M}_{\mathfrak{a}} \mathfrak{b}^{j}} .
$$

If we take $\mathcal{M}=\hat{M}_{\mathfrak{a}} / \hat{M}_{\mathfrak{a}} \mathfrak{b}^{j}$, then (5.4) implies

$$
\lim _{i} \frac{\hat{M}_{\mathfrak{a}}}{\hat{M}_{\mathfrak{a}} \mathfrak{a}^{i}+\hat{M}_{\mathfrak{a}} \mathfrak{b}^{j}}=\hat{M}_{\mathfrak{a}} / \hat{M}_{\mathfrak{a}} \mathfrak{b}^{j} .
$$

Therefore we have

$$
\lim _{j}{\underset{\lim }{\leftarrow}}_{i} \frac{M}{M \mathfrak{a}^{i}+M \mathfrak{b}^{j}}=\left(\hat{M}_{\mathfrak{a}}\right)_{\mathfrak{b}} .
$$

Using that a double inverse limit is the limit over the ordered set $\mathbb{N}^{2}$ and that the diagonal $\{(i, i) \mid i \in \mathbb{N}\}$ is a cofinal subset, we obtain

$$
\lim _{i} \frac{M}{M \mathfrak{a}^{i}+M \mathfrak{b}^{i}}=\left(\hat{M}_{\mathfrak{a}}\right)_{\mathfrak{b}}^{\wedge}
$$

Finally, using that $M(\mathfrak{a}+\mathfrak{b})^{2 i} \subseteq M \mathfrak{a}^{i}+M \mathfrak{b}^{i} \subseteq M(\mathfrak{a}+\mathfrak{b})^{i}$, we obtain $\hat{M}_{\mathfrak{a}+\mathfrak{b}}=\left(\hat{M}_{\mathfrak{a}}\right)_{\mathfrak{b}}$.

Let $A$ be a finitely generated abelian group and $\Lambda$ be a Noetherian commutative ring. Then the group ring $\Lambda[A]$ is a Noetherian commutative ring. We denote by $I_{\Lambda}$ the augmentation ideal of $\Lambda[A]$, denote by $p \cdot \Lambda[A]$ the ideal of $\mathbb{Z}[A]$ generated by $p \in \Lambda \subset \Lambda[A]$ and set

$$
I_{p, \Lambda}:=I_{\Lambda}+p \cdot \Lambda[A]=\operatorname{Ker}(\Lambda[A] \rightarrow \Lambda / p) .
$$

Moreover, we set

$$
I=I_{\mathbb{Z}}, \quad I_{p}=I_{p, \mathbb{Z}} .
$$

It is easy to see that, if $M$ is a $\Lambda[A]$-module, then its $\mathbb{Z} / p$-completion as an abelian group coincides with the $p \cdot \Lambda[A]$-adic completion

$$
\hat{M}_{\mathbb{Z} / p}=\hat{M}_{p \cdot \Lambda[A]} .
$$

In particular, it does not depend on $\Lambda$. Moreover, if $A$ is generated by $t_{1}, \ldots, t_{n}$, then $I_{\Lambda}=$ $\sum \Lambda[A]\left(t_{i}-1\right)$ and

$$
M I_{\Lambda}=\sum M\left(t_{i}-1\right)=M I, \quad M I_{p, \Lambda}=M I+p M=M I_{p} .
$$

It follows that the completions do not depend on $\Lambda$

$$
\hat{M}_{I_{\Lambda}}=\hat{M}_{I}, \quad \hat{M}_{I_{p, \Lambda}}=\hat{M}_{I_{p}}
$$

and we can use the notations $\hat{M}_{I}$ and $\hat{M}_{I_{p}}$ for any $\Lambda$. 
Corollary 5.2. Let $A$ be a finitely generated abelian group, $\Lambda$ be a Noetherian commutative ring and $M$ be a finitely generated $\Lambda[A]$-module. Then

$$
\left(\hat{M}_{\mathbb{Z} / p}\right)_{I}^{\wedge}=\hat{M}_{I_{p}}=\left(\hat{M}_{I}\right)_{\mathbb{Z} / p}^{\wedge}
$$

By $\mathbb{Z}_{p}$ we denote the ring of $p$-adic integers $\mathbb{Z}_{p}=\lim _{\longleftarrow} \mathbb{Z} / p^{i}=\hat{\mathbb{Z}}_{\mathbb{Z} / p}$.

Corollary 5.3. Let $A$ be a finitely generated abelian group and $M$ be a $\mathbb{Z}_{p}[A]$-module, which is finitely generated over $\mathbb{Z}_{p}$. Then

$$
\lim _{\longleftarrow}\left(M / p^{i}\right)_{I}^{\wedge}=\hat{M}_{I}=\hat{M}_{I_{p}}
$$

Proof. It follows from the equation $\left(M / p^{i}\right)_{I}^{\wedge}=\left(\mathbb{Z} / p^{i} \otimes M\right) \otimes_{\mathbb{Z}_{p}[A]} \overline{\mathbb{Z}} p[A]_{I}=\mathbb{Z} / p^{i} \otimes\left(M \otimes_{\mathbb{Z}_{p}[A]}\right.$ $\left.\overline{\mathbb{Z}}_{p}[A]_{I}\right)=\left(\hat{M}_{I}\right) / p^{i}$, the equation $\hat{M}_{\mathbb{Z} / p}=M$ and Corollary 5.2 .

By $C$ we denote the infinite cyclic group.

Lemma 5.4. Let $\mathcal{K}$ be an Artinian commutative ring and $M$ be a $\mathcal{K}[C]$-module, which is finitely generated over $\mathcal{K}$. Then the $C$-module $\hat{M}_{I}$ is nilpotent, the homomorphism $M \rightarrow \hat{M}_{I}$ is a split homomorphism of $C$-modules and there is an isomorphism

$$
M=M I^{\infty} \oplus \hat{M}_{I},
$$

which is natural by $M$, where $M I^{\infty}=\bigcap_{i=1}^{\infty} M I^{i}$.

Proof. We denote by $a: M \rightarrow M$ the multiplication on $t$ and set $b:=a-1$. Then there is positive integer $n$ such that $\operatorname{Im}\left(b^{n}\right)=\operatorname{Im}\left(b^{n+1}\right), \operatorname{Ker}\left(b^{n}\right)=\operatorname{Ker}\left(b^{n+1}\right)$ and $V=\operatorname{Im}\left(b^{n}\right) \oplus \operatorname{Ker}\left(b^{n}\right)$ [14, Cor. 6.4.2]. Moreover, it is easy to see that $M I^{i}=\operatorname{Im}\left(b^{i}\right)$. It follows that $M I^{i}=\operatorname{Im}\left(b^{n}\right)$ for $i \geq n, \hat{V}_{I}=\operatorname{Ker}\left(b^{n}\right)$ and $V \cong \hat{V}_{I} \oplus V I^{i}$.

Lemma 5.5. Let $\mathcal{M}$ be a $\mathbb{Z}_{p}[C]$-module, which is finitely generated over $\mathbb{Z}_{p}$. Then $\mathcal{M}_{I}=$ $\mathcal{M}_{I_{p}}$ the homomorphism $\mathcal{M} \rightarrow \hat{\mathcal{M}}_{I}$ splits in the category of $C$-modules and there is a natural isomorphism

$$
\mathcal{M}=\mathcal{M} I^{\infty} \oplus \hat{\mathcal{M}}_{I}
$$

where $\mathcal{M} I^{\infty}=\cap \mathcal{M} I^{i}$. Moreover, if $C=\langle t\rangle$ and $\mathcal{M}(t-1) \subseteq p \mathcal{M}$, then $\mathcal{M} \cong \hat{\mathcal{M}}_{I}$.

Proof. Since $\mathcal{M}$ is a finitely generated $\mathbb{Z}_{p}$-module, $\mathcal{M}$ is isomorphic to $\mathbb{Z}_{p}^{n} \oplus \bigoplus_{j=1}^{m} \mathbb{Z} / p^{k_{j}}$ as an abelian group. The quotient $\mathcal{M} / p^{i}$ is a $\mathbb{Z} / p^{i}[C]$-module, which is finitely generated over $\mathbb{Z} / p^{i}$. It follows that the map $\mathcal{M} / p^{i} \rightarrow\left(\mathcal{M} / p^{i}\right)_{I}^{\wedge}$ is a split epimorphism of $\mathbb{Z} / p^{i}[C]$-modules. Denote the section by $s_{i}:\left(\mathcal{M} / p^{i}\right)_{I}^{\wedge} \rightarrow \mathcal{M} / p^{i}$. Moreover, using that the section is natural, we obtain that the diagram

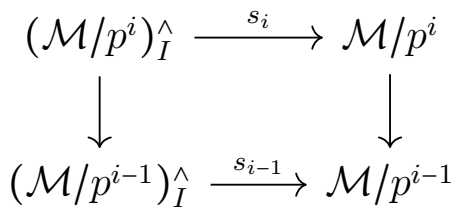

is commutative. It follows that $\left\{s_{i}\right\}$ induce a section on the level of inverse limits. Using Corollary 5.3, we obtain that $\mathcal{M} \rightarrow \hat{\mathcal{M}}_{I}$ is a split epimorphism of $C$-modules and the section is natural. The kernel of the epimorphism equals to $\mathcal{M} I^{\infty}$. Then $\mathcal{M}=\mathcal{M} I^{\infty} \oplus \hat{\mathcal{M}}_{I}$. If $\mathcal{M} I=$ $\mathcal{M}(t-1) \subseteq p \mathcal{M}$, then $\mathcal{M} I^{i} \subseteq p^{i} \mathcal{M}$, and hence, $\mathcal{M} I^{\infty}=0$ and $\mathcal{M}=\hat{\mathcal{M}}_{I}$. 


\subsection{Completion of metabelian groups over $C$.}

Lemma 5.6. Let $G$ be a group, $N$ be a nilpotent $G$-module and $X$ be an abelian group. Then $H_{n}(N, X)$ is a nilpotent $G$-module.

Proof. Let $N$ be a nilpotent $G$-module of class $m$. The proof is by induction on $m$. If $m=1$, then $N$ is a trivial $G$-module, and hence, $H_{n}(N, X)$ is trivial. Prove the inductive step. Consider a short exact sequence $T \nrightarrow N \rightarrow N^{\prime}$, where $T$ is a trivial module and $N^{\prime}$ is a nilpotent module of class $m-1$. By inductive hypothesis the second page of the spectral sequence consists of nilpotent modules $H_{i}\left(N^{\prime}, H_{j}(T, X)\right)$. It follows that $H_{n}(N, X)$ is nilpotent.

By $C$ we denote the infinite cyclic group. A group $G$ is said to be metabelian over $C$ if there is a short exact sequence $M \nrightarrow G \rightarrow C$, where $M$ is abelian. Then $M$ can be considered as a $C$-module, where the action of $C$ is induced by the conjugation. It is easy to see that $G=M \rtimes C$. Hence the group structure on $G$ depends only on the $C$-module $M$. Then the completions can be described as follows

$$
\hat{G}_{\mathbb{Z}}=\hat{M}_{I} \rtimes C, \quad \hat{G}_{\mathbb{Z} / p}=\hat{M}_{I_{p}} \rtimes \mathbb{Z}_{p}, \quad \hat{G}_{\mathbb{Q}}=(M \otimes \mathbb{Q})_{I}^{\wedge} \rtimes \mathbb{Q},
$$

where $I$ is the augmentation ideal of $\mathbb{Z}[C]$ and $I_{p}=I+p \cdot \mathbb{Z}[C]$ (see Prop. 4.7 and Prop. 4.12 of [13]).

Proposition 5.7. Let $M$ be a $C$-module such that $M \otimes \mathbb{Q}$ is finite dimensional and $G=M \rtimes C$. Then the map

$$
H_{n}(G, \mathbb{Q}) \rightarrow H_{n}\left(\hat{G}_{\mathbb{Q}}, \mathbb{Q}\right)
$$

is an epimorphism for any $n$.

Proof. Set $V=M \otimes \mathbb{Q}$. The homomorphism $G \rightarrow \hat{G}_{\mathbb{Q}}$ induces a morphism of the spectral sequences

$$
H_{i}\left(C, H_{j}(M, \mathbb{Q})\right) \longrightarrow H_{i}\left(\mathbb{Q}, H_{j}\left(\hat{V}_{I}, \mathbb{Q}\right)\right) .
$$

Note that $H_{i}(C,-)=0$ and $H_{i}(\mathbb{Q},-)=0$ for $i \geq 2$. Indeed, for $C$ it is obvious and for $\mathbb{Q}$ it follows from the fact that homology commutes with direct limits $\left(H_{*}\left(\lim _{\longrightarrow} G_{i}, M\right)=\underline{\lim } H_{*}\left(G_{i}, M\right)\right)$ and $\mathbb{Q}=\underset{\lim }{\longrightarrow}$. Then the spectral sequences have only two nontrivial columns and the morphism of spectral sequences gives a morphism of the short exact sequences

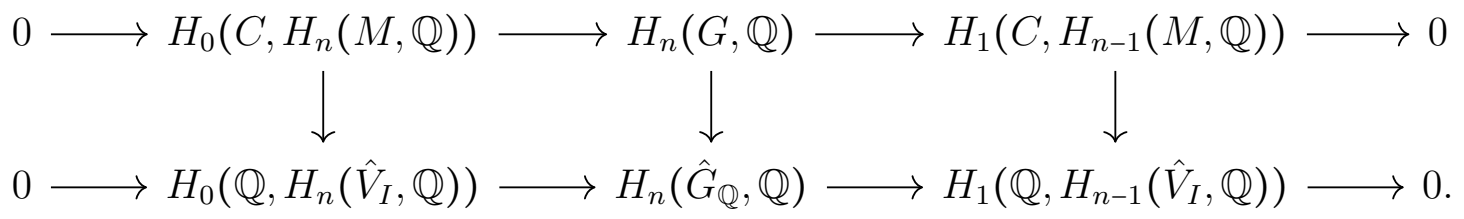

Therefore, it is enough to prove that

$$
H_{i}\left(C, H_{n}(M, \mathbb{Q})\right) \longrightarrow H_{i}\left(\mathbb{Q}, H_{n}\left(\hat{V}_{I}, \mathbb{Q}\right)\right)
$$

is an epimorphism for any $n$ and $i=0,1$.

Note that $H_{*}(C, \mathbb{Q})=H_{*}(\mathbb{Q}, \mathbb{Q})$. Using this and the long exact sequence associated with a short exact sequence of modules, we obtain

$$
H_{*}(C, \mathcal{N})=H_{*}(\mathbb{Q}, \mathcal{N})
$$


for any nilpotent $\mathbb{Q}[\mathbb{Q}]$-module $\mathcal{N}$. If $\mathcal{N}$ is a nilpotent $\mathbb{Q}[C]$-module, there is a unique way to lift the action of $C=\langle t\rangle$ to the action of $\mathbb{Q}=\left\{t^{\alpha} \mid \alpha \in \mathbb{Q}\right\}$ on $\mathcal{N}$ :

$$
x t^{\alpha}=\sum_{n=0}^{\infty}\left(\begin{array}{l}
\alpha \\
n
\end{array}\right) x(t-1)^{n}
$$

where $\alpha \in \mathbb{Q}, x \in \mathcal{N}$ (see Lemma 4.4 of [13]). The sum is finite because $\mathcal{N}(t-1)^{n}=0$ for big enough $n$. It is easy to see from the formula that $\mathcal{N}\left(t^{\alpha}-1\right) \subseteq \mathcal{N}(t-1)$. It follows that the $\mathbb{Q}[\mathbb{Q}]$-module $\mathcal{N}$ is nilpotent if and only if it is nilpotent as a $\mathbb{Q}[C]$-module.

The module $\hat{V}_{I}$ is nilpotent as a $\mathbb{Q}[C]$-module (Lemma 5.4). Then $H_{n}\left(\hat{V}_{I}, \mathbb{Q}\right)$ is nilpotent as $\mathbb{Q}[C]$-module (Lemma [5.6), and hence, it is nilpotent as a $\mathbb{Q}[\mathbb{Q}]$-module. It follows that

$$
H_{i}\left(C, H_{n}\left(\hat{V}_{I}, \mathbb{Q}\right)\right)=H_{i}\left(\mathbb{Q}, H_{n}\left(\hat{V}_{I}, \mathbb{Q}\right)\right) .
$$

Hence it is sufficient to prove that the homomorphism

$$
H_{n}(M, \mathbb{Q}) \longrightarrow H_{n}\left(\hat{V}_{I}, \mathbb{Q}\right)
$$

is a split epimorphism in the category of $C$-modules. Note that $H_{n}(M, \mathbb{Q})=\Lambda^{n}(V)$ and $H_{n}\left(\hat{V}_{I}, \mathbb{Q}\right)=\Lambda^{n}\left(\hat{V}_{I}\right)$. Then the assertion follows from the fact that $V \rightarrow \hat{V}_{I}$ is a split epimorphism of $C$-modules (Lemma 5.4).

Theorem 5.8. Let $M$ be a finitely generated $C$-module such that $\operatorname{tor}(M)$ is finite and $M \otimes \mathbb{Q}$ is finite dimensional. Set $G=M \rtimes C$. Then

$$
H_{*}\left(\hat{G}_{\mathbb{Z}}, \mathbb{Z} / p\right)=H_{*}\left(\hat{G}_{\mathbb{Z} / p}, \mathbb{Z} / p\right)
$$

and the morphism

$$
H_{n}(G, \mathbb{Z} / p) \longrightarrow H_{n}\left(\hat{G}_{\mathbb{Z} / p}, \mathbb{Z} / p\right)
$$

is an epimorphism for any $n$. Moreover, if $C=\langle t\rangle$ and $M(t-1) \subseteq p M$, then

$$
H_{*}(G, \mathbb{Z} / p) \cong H_{*}\left(\hat{G}_{\mathbb{Z} / p}, \mathbb{Z} / p\right) \quad \text { and } \quad(B G)_{\mathbb{Z} / p} \cong B\left(\hat{G}_{\mathbb{Z} / p}\right) .
$$

Proof. Set $\mathcal{M}=\hat{M}_{\mathbb{Z} / p}$. Then $\mathcal{M}$ is a finitely generated $\mathbb{Z}_{p}$-module (Proposition 4.10) and it is isomorphic to $\mathbb{Z}_{p}^{m} \oplus \bigoplus_{j=1}^{m} \mathbb{Z} / p^{k_{j}}$ as an abelian group. Using Corollary 5.2 and Lemma 5.5 we obtain

It follows that

$$
\hat{\mathcal{M}}_{I}=\hat{\mathcal{M}}_{I_{p}}=\left(\hat{M}_{I}\right)_{\mathbb{Z} / p}^{\wedge}=\hat{M}_{I_{p}}, \quad \mathcal{M}=\mathcal{M} I^{\infty} \oplus \hat{\mathcal{M}}_{I}
$$

$$
H_{*}(\mathcal{M}, \mathbb{Z} / p) \longrightarrow H_{*}\left(\hat{\mathcal{M}}_{I}, \mathbb{Z} / p\right)
$$

is a split epimorphism of $C$-modules. By Proposition 4.10 we obtain $H_{*}(\mathcal{M}, \mathbb{Z} / p)=$ $H_{*}(M, \mathbb{Z} / p)$ and $H_{*}\left(\hat{M}_{I_{p}}, \mathbb{Z} / p\right)=H_{*}\left(\hat{\mathcal{M}}_{I}, \mathbb{Z} / p\right)=H_{*}\left(\hat{M}_{I}, \mathbb{Z} / p\right)$. It follows that

$$
H_{*}(M, \mathbb{Z} / p) \longrightarrow H_{*}\left(\hat{M}_{I}, \mathbb{Z} / p\right)
$$

is a split epimorphism of $C$-modules and

$$
H_{*}\left(\hat{M}_{I}, \mathbb{Z} / p\right)=H_{*}\left(\hat{M}_{I_{p}}, \mathbb{Z} / p\right) .
$$

By Theorem 3.11 we get that $H_{n}\left(\hat{M}_{I_{p}}, \mathbb{Z} / p\right)$ is a finite $\mathbb{Z} / p\left[\mathbb{Z}_{p}\right]$-module. Using Proposition 4.10, we obtain

$$
H_{*}\left(C, H_{n}\left(\hat{M}_{I}, \mathbb{Z} / p\right)\right)=H_{*}\left(C, H_{n}\left(\hat{M}_{I_{p}}, \mathbb{Z} / p\right)\right)=H_{\star}\left(\mathbb{Z}_{p}, H_{n}\left(\hat{M}_{I_{p}}, \mathbb{Z} / p\right)\right) .
$$

Since $\hat{G}_{\mathbb{Z}}=\hat{M}_{I} \rtimes C$ and $\hat{G}_{\mathbb{Z} / p}=\hat{M}_{I_{p}} \rtimes \mathbb{Z}_{p}$, it follows that

$$
H_{*}\left(\hat{G}_{\mathbb{Z}}, \mathbb{Z} / p\right)=H_{*}\left(\hat{G}_{\mathbb{Z} / p}, \mathbb{Z} / p\right) .
$$


The homomorphism $G \rightarrow \hat{G}_{\mathbb{Z}}$ induces a morphism of the spectral sequences

$$
H_{i}\left(C, H_{j}(M, \mathbb{Z} / p)\right) \longrightarrow H_{i}\left(C, H_{j}\left(\hat{M}_{I}, \mathbb{Z} / p\right)\right)
$$

The spectral sequences have only two nontrivial columns and the morphism of spectral sequences gives a morphism of the short exact sequences

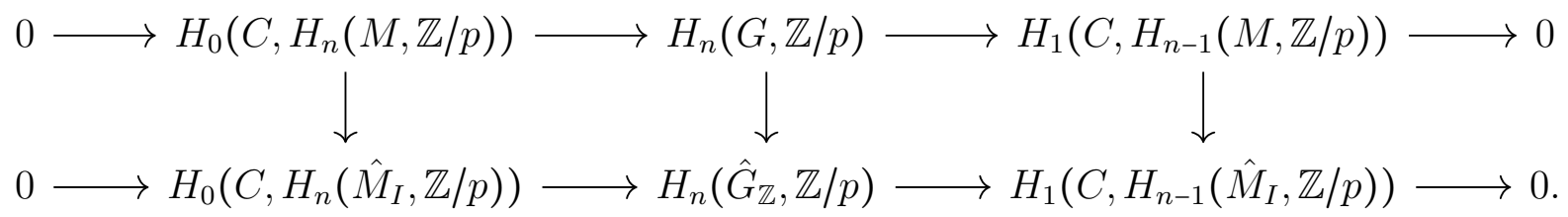

Using that the left and the right vertical arrows are split epimorphisms, we obtain that the middle vertical arrow is an epimorphism.

Assume now $M(t-1) \subseteq p M$. Then $\mathcal{M}(t-1) \subseteq p \mathcal{M}$. Hence, $\mathcal{M} I^{\infty}=0$. It follows that the map (5.6) is an isomorphism. Then (5.7) is an isomorphism. Then the left and the right vertical arrows in the diagram (5.8) are isomorphisms. Then $H_{*}(G, \mathbb{Z} / p)=H_{*}\left(\hat{G}_{\mathbb{Z}}, \mathbb{Z} / p\right)=$ $H_{*}\left(\hat{G}_{\mathbb{Z} / p}, \mathbb{Z} / p\right)$. Then $B G \rightarrow B\left(\hat{G}_{\mathbb{Z} / p}\right)$ is a $\mathbb{Z} / p$-homological equivalence. Moreover, the space $B\left(\hat{G}_{\mathbb{Z} / p}\right)$ is $\mathbb{Z} / p$-local because the group $\hat{G}_{Z Z / p}$ is $H \mathbb{Z} / p$-local. It follows that $(B G)_{\mathbb{Z} / p}=$ $B\left(\hat{G}_{\mathbb{Z} / p}\right)$.

Corollary 5.9. Let $M$ be a $C$-module such that $M \rtimes C$ is finitely presented. Set $G=M \rtimes C$. Then

and the morphism

$$
H_{\star}\left(\hat{G}_{\mathbb{Z}}, \mathbb{Z} / p\right)=H_{\star}\left(\hat{G}_{\mathbb{Z} / p}, \mathbb{Z} / p\right)
$$

$$
H_{n}(G, \mathbb{Z} / p) \longrightarrow H_{n}\left(\hat{G}_{\mathbb{Z} / p}, \mathbb{Z} / p\right)
$$

is an epimorphism for any $n$. Moreover, if $C=\langle t\rangle$ and $M(t-1) \subseteq p M$, then

$$
H_{*}(G, \mathbb{Z} / p) \cong H_{*}\left(\hat{G}_{\mathbb{Z} / p}, \mathbb{Z} / p\right) \quad \text { and } \quad(B G)_{\mathbb{Z} / p} \cong B\left(\hat{G}_{\mathbb{Z} / p}\right) .
$$

Corollary 5.10. Let $a \in \mathrm{GL}_{n}(\mathbb{Z})$ such that entries of $a-1$ are divisible by $p$. Set $G=\mathbb{Z}^{n} \rtimes_{a} C$. Then $(B G)_{\mathbb{Z} / p}=B\left(\hat{G}_{\mathbb{Z} / p}\right)$.

\section{REFERENCES}

[1] H. Abels: An example of a finitely presented solvable group, Homological group theory (Proc. Sympos., Durham, 1977), London Math. Soc. Lecture Note Ser., vol. 36, Cambridge Univ. Press, Cambridge, 1979, pp. 205-211.

[2] H. Åberg: Bieri-Strebel valuations (of finite rank), Proc. London. Math. Soc. (3) 52 (1986), pp. 269-304.

[3] R. Bieri and K. Strebel: Almost finitely presented soluble groups, Comm. Math. Helv. 53 (1978), pp. 258-278.

[4] R. Bieri and R. Strebel: Valuations and finitely presented metabelian groups, Proc. London Math. Soc. (3) 41 (1980), pp. 439-464.

[5] N. Bourbaki, Commutative Algebra, Addison-Wesley, Paris, 1972.

[6] A.K. Bousfield: The localization of spaces with respect to homology, Topology Volume 14, Issue 2, June 1975, pp. 133-150.

[7] A. K. Bousfield: Homological localization towers for groups and $\pi$-modules, Mem. Amer. Math. Soc, no. $186,1977$.

[8] A. K. Bousfield, On the p-Adic Completions of Nonnilpotent Spaces, Transactions of the American Mathematical Society Vol. 331, No. 1 (May, 1992), pp. 335-359.

[9] K. Brown, Cohomology of Groups, Springer-Verlag GTM 87, 1982. 
[10] H. Cartan et al.: Algébre d'Eilenberg-Maclane et homotopie: seminaire, 7e annee, École normale supérieure (Paris), 1954/1955.

[11] L. Fuchs: Infinite abelian groups, Academic Press, New York and London.

[12] P. Hilton, Localization and cohomology of nilpotent groups, Math. Z. 132 (1973), pp. 263-286.

[13] S.O. Ivanov, R. Mikhailov: On a problem of Bousfield for metabelian groups, Advances in Mathematics, Volume 290 (2016), pp. 552-589.

[14] F. Kash: Modules and Rings, Academic Press (1982).

[15] D.J.S. Robinson: Finiteness condition and generalized soluble groups, Part 1, Springer (1972).

[16] L. Ribes, P. Zalesskii: Profinite Groups, A Series of Modern Surveys in Mathematics, Volume 40 (2010).

[17] J.D. Stasheff: Continuous cohomoloyg of groups and classifying spaces, Bull. Amer. Math. Soc. 84 (1978), pp. 513-530.

Chebyshev Laboratory, St. Petersburg State University, 14th Line, 29b, Saint Petersburg, 199178 RUSSIA

E-mail address: ivanov.s.o.1986@gmail.com 